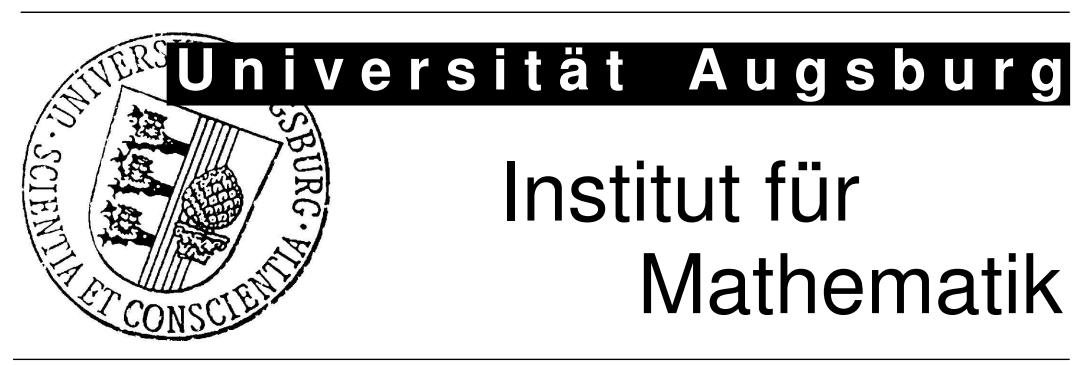

Ronald H.W. Hoppe; Christopher Linsenmann

An Adaptive Newton Continuation Strategy for the Fully Implicit Finite Element Immersed Boundary Method 


\section{Impressum:}

\section{Herausgeber:}

Institut für Mathematik

Universität Augsburg

86135 Augsburg

http://www.math.uni-augsburg.de/pages/de/forschung/preprints.shtml

\section{ViSdP:}

Ronald H.W. Hoppe

Institut für Mathematik

Universität Augsburg

86135 Augsburg

Preprint: Sämtliche Rechte verbleiben den Autoren (C) 2011 


\title{
AN ADAPTIVE NEWTON CONTINUATION STRATEGY \\ FOR THE FULLY IMPLICIT FINITE ELEMENT IMMERSED BOUNDARY METHOD
}

\author{
R.H.W. HOPPE ${ }^{\dagger \ddagger \S}$ AND C. LINSENMANN ${ }^{\dagger}$
}

\begin{abstract}
The Immersed Boundary Method (IB) is known as a powerful technique for the numerical solution of fluid-structure interaction problems as, for instance, the motion and deformation of viscoelastic bodies immersed in an external flow. It is based on the treatment of the flow equations within an Eulerian framework and of the equations of motion of the immersed bodies with respect to a Lagrangian coordinate system including interaction equations providing the transfer between both frames. The classical IB uses finite differences, but the IBM can be set up within a finite element approach in the spatial variables as well (FE-IB). The discretization in time usually relies on the Backward Euler (BE) method for the semidiscretized flow equations and the Forward Euler (FE) method for the equations of motion of the immersed bodies. The BE/FE FE-IB is subject to a CFL-type condition, whereas the fully implicit BE/BE FE-IB is unconditionally stable. The latter one can be solved numerically by Newton-type methods whose convergence properties are dictated by an appropriate choice of the time step size, in particular, if one is faced with sudden changes in the total energy of the system. In this paper, taking advantage of the well developed affine covariant convergence theory for Newton-type methods, we study a predictor-corrector continuation strategy in time with an adaptive choice of the continuation steplength. The feasibility of the approach and its superiority to BE/FE FE-IB is illustrated by a representative numerical example.
\end{abstract}

Key words. finite element immersed boundary method, fully implicit scheme, predictorcorrector continuation, red blood cells

AMS subject classifications. 65H20, 65M60, 74L15, 76D05, 92C10

1. Introduction. A computationally attractive methodology for the numerical simulation of the motion and deformation of elastic and viscoelastic bodies in external flows is the Immersed Boundary Method (IB), which has been originally developed by Peskin [28] and further studied in [11, 29, 30,31,33]. The IBM uses an Eulerian coordinate system for the flow equations and Lagrangian coordinates for the boundary of the immersed bodies together with appropriate interaction equations to transform Eulerian to Lagrangian quantities and vice versa. The interaction equations feature multidimensional Dirac delta functions that have to be approximated appropriately within a finite difference approach. More recently, a variational formulation of the IBM has been provided in [8] and $[9,10]$ as a basis for a finite element realization referred to as the Finite Element Immersed Boundary Method (FE-IB). Both for the classical IB and the FE-IB, the most common approach with regard to discretization in time is to use the Backward Euler (BE) method for the flow equations and the Forward Euler (FE) method for the equation describing the motion and deformation of the immersed bodies which gives rise to the $\mathrm{BE} / \mathrm{FE} \mathrm{IB}$ and $\mathrm{BE} / \mathrm{FE} \mathrm{FE}-\mathrm{IB}$, respectively. However, these schemes typically require a CFL-type condition (cf., e.g., $[9,16])$. Better stability properties can be achieved by using the BE method as a time integrator for both equations thus resulting in a fully implicit scheme. Recent

\footnotetext{
$\dagger$ Dept. of Math., Univ. of Houston, Houston, TX 77204-3008, U.S.A.

$\ddagger$ Inst. of Math., Univ. of Augsburg, D-86159 Augsburg, Germany

§Supported by NSF grants DMS-0707602, DMS-0810176, DMS-0811153, DMS-0914788, by the DFG within the Priority Programs SPP 1253 and SPP 1506, by the BMBF within the Collaborative Research Projects 'FROPT' and 'MeFreSim', and by the ESF within the Research Networking Programme 'OPTPDE'.

${ }^{\top}$ Supported by the DFG within the Priority Program SPP 1253.
} 
applications of the $\mathrm{BE} / \mathrm{BE} \mathrm{IB}$ can be found in $[23,24,25]$, whereas the unconditional stability of the BE/BE FE-IB has been shown in [9]. For each time step, the implementation of the fully implicit scheme requires the solution of a nonlinear system of equations for which a Newton-type method would be the method of choice using the result of the previous step as an initial guess. Sudden changes in the energy of the system, for instance due to large deformations of the immersed bodies, may lead to significant restrictions of the time step size in order to guarantee convergence of the Newton-type iteration. Such scenarios can be handled adequately by an adaptive choice of the time step size which can be realized by treating the problem as a parameter dependent one and using a predictor-corrector continuation in time based on the affine covariant convergence theory of Newton-type methods (cf., e.g., [15] and the references therein). It is the purpose of this contribution to provide such an adaptive continuation strategy for the fully implicit FE-IB. The paper is organized as follows: In section 2, we begin with a brief overview on the FE-IB which is based on the variational formulation of the problem (subsection 2.1) and then derive the fully implicit scheme giving rise to the solution of a nonlinear system of equations at each time step (subsection 2.2). In particular, we show that the associated nonlinear map admits an invertible Jacobian under some constraints on the time step size (Theorem 2.3) and discuss scenarios for which we may encounter severe time step restrictions. Section 3 is devoted to the adaptive continuation method which is a predictor-corrector continuation method in time. It uses classical continuation as a predictor and a combination of the ordinary and the simplified Newton method as a corrector featuring an adaptive choice of the continuation steplength based on information of the previous successful continuation step and a monotonicity test for convergence monitoring. In section 4 , we provide a representative example which is the motion and deformation of a red blood cell through a capillary within a microfluidic channel flow. We address the shortcomings of the BE/FE FE-IB and illustrate the superior performance of the adaptive continuation approach based on the fully implicit scheme.

2. Finite Element Immersed Boundary Method. We consider the motion and deformation of viscoelastic bodies such as vesicles and red blood cells immersed in an incompressible external fluid. The classical IB as developed in [28] uses three groups of equations:

- the incompressible Navier-Stokes equations describing the motion of the fluid within an Eulerian coordinate system,

- the material elasticity equations describing the deformation of the immersed bodies in terms of the change of the total elastic energy within a Lagrangian coordinate system,

- the interaction equations which transform Eulerian into Lagrangian quantities and vice versa.

The FE-IB has been originally studied in [8] and [9]. It relies on the variational formulation of these equations which will be addressed in subsection 2.1. The fully implicit FE-IB which will be dealt with in subsection 2.2 is based on finite element approximations of the variational equations in space and implicit time discretizations by the Backward Euler (BE) scheme both for the semi-discretized Navier-Stokes and interaction equations. Therefore, it will be referred to as the BE/BE FE-IB.

2.1. Variational formulation of the IB. We assume $\Omega=(a, b) \times(c, d), a<$ $b, c<d$, to be a domain in $\mathbb{R}^{2}$ with boundary $\Gamma=\bar{\Gamma}_{\text {in }} \cup \bar{\Gamma}_{\text {lat }} \cup \bar{\Gamma}_{\text {out }}$, where $\Gamma_{\text {in }}:=$ $\{a\} \times(c, d), \Gamma_{\text {out }}:=\{b\} \times(c, d)$, and $\Gamma_{\text {lat }}:=\Gamma_{\text {bot }} \cup \Gamma_{\text {top }}, \Gamma_{\text {bot }}:=(a, b) \times\{c\}, \Gamma_{\text {top }}:=$ $(a, b) \times\{d\}$. We set $Q:=\Omega \times(0, T), \Sigma_{\text {in }}:=\Gamma_{\text {in }} \times(0, T), \Sigma_{\text {lat }}:=\Gamma_{\text {lat }} \times(0, T), \Sigma_{\text {out }}:=$ 
$\Gamma_{\text {out }} \times(0, T)$, where $T>0$. We assume that the fluids has density $\rho>0$ and viscosity $\nu>0$. We denote by $\mathbf{u}=\mathbf{u}(x, t)$ and $p=p(x, t),(x, t) \in Q$, the velocity and the pressure. We further refer to $\varepsilon(\mathbf{u}):=\left(\nabla \mathbf{u}+(\nabla \mathbf{u})^{T}\right) / 2$ as the linearized strain tensor and to $\boldsymbol{\sigma}(\mathbf{u}, p):=-p \mathbf{I}+2 \nu \boldsymbol{\varepsilon}(\mathbf{u})$ as the stress tensor. Assuming a force density $\mathbf{F}$ in $Q$, periodic boundary conditions in terms of a prescribed stationary velocity $\mathbf{g}$ at the inflow boundary $\Sigma_{\text {in }}$ and the outflow boundary $\Sigma_{\text {out }}$, zero velocity on $\Sigma_{\text {lat }}$, and an initial velocity $\mathbf{u}^{(0)}$ at time $t=0$, the incompressible Navier Stokes equations read

$$
\begin{aligned}
\rho\left(\frac{\partial \mathbf{u}}{\partial t}+(\mathbf{u} \cdot \nabla) \mathbf{u}\right)-\nu \Delta \mathbf{u}+\nabla p & =\mathbf{F} \text { in } Q, \\
\nabla \cdot \mathbf{u} & =0 \text { in } Q, \\
\mathbf{u}=\mathbf{g}=(g, 0)^{T}, g \geq 0 \text { on } \Sigma^{\prime}, \Sigma^{\prime} \in & \left\{\Sigma_{\text {in }}, \Sigma_{\text {out }}\right\}, \\
\mathbf{u} & =\mathbf{0} \text { on } \Sigma_{\text {lat }}, \\
\mathbf{u}(\cdot, 0) & =\mathbf{u}^{(0)} \text { in } \Omega .
\end{aligned}
$$

For the variational formulation of (2.1a)-(2.1e) we use standard notation from Lebesgue and Sobolev space theory (cf., e.g., $[18,36]$ ). In particular, for a bounded domain $\Omega \subset \mathbb{R}^{d}, d \in \mathbb{N}$, we denote by $L^{p}(\Omega)$ and $\mathbf{L}^{p}(\Omega):=L^{p}(\Omega)^{d}, 1 \leq p \leq \infty$, the Banach space of to the power $p$ square integrable scalar- and vector-valued functions on $\Omega$. In particular, for $p=2$ the spaces $L^{2}(\Omega)$ and $\mathbf{L}^{2}(\Omega)$ are Hilbert spaces, equipped with the inner product $(\cdot, \cdot)_{0, \Omega}$ and the associated norm $\|\cdot\|_{0, \Omega} \cdot L_{0}^{2}(\Omega)$ stands for the subspace of functions with zero integral mean. Further, we denote by $\mathbf{H}^{s}(\Omega), s \in \mathbb{R}_{+}$, the Sobolev space of vector-valued functions with the inner product $(\cdot, \cdot)_{s, \Omega}$ and the associated norm $\|\cdot\|_{s, \Omega}$. The space $\mathbf{H}_{0, \Gamma^{\prime}}^{s}(\Omega)$ is the subspace with vanishing trace on $\Gamma^{\prime} \subseteq \Gamma$. We will omit the subindex $\Gamma^{\prime}$, if $\Gamma^{\prime}=\Gamma . \mathbf{H}^{-s}(\Omega)$ stands for the dual space of $\mathbf{H}_{0}^{s}(\Omega)$ with $\langle\cdot, \cdot\rangle$ referring to the dual product. The space $\mathbf{H}^{s}(\bar{\Omega}) \subset \mathbf{H}^{s}(\Omega)$ is the subspace of all $\left.\mathbf{u}\right|_{\Omega}$ where $\mathbf{u} \in \mathbf{H}^{s}\left(\mathbb{R}^{d}\right)$ and $\left\langle\left.\mathbf{u}\right|_{\Omega}, \boldsymbol{\varphi}\right\rangle=\langle\mathbf{u}, \tilde{\varphi}\rangle$ for all $\boldsymbol{\varphi} \in \mathbf{C}_{0}^{\infty}(\Omega)$ with $\tilde{\varphi}$ referring to the continuation of $\varphi$ by zero outside $\Omega$. We denote by $\mathbf{H}^{s-1 / 2}\left(\Gamma^{\prime}\right), s \geq 1$, the trace space of vector-valued functions on $\Gamma^{\prime}$. We further refer to $\mathbf{H}_{00}^{s-1 / 2}\left(\Gamma^{\prime}\right)$ as the space of functions whose extensions by zero to $\Gamma \backslash \Gamma^{\prime}$ belong to $\mathbf{H}^{s-1 / 2}(\Gamma)$. Finally, we denote by $C^{k}(\Omega)$ and $\mathbf{C}^{k}(\Omega), k \in \mathbb{N}_{0}$ the Banach spaces of $k$-times continuously differentiable scalar- and vector-valued functions on $\Omega$.

Moreover, for $T>0$ and a Banach space $Z(\mathbf{Z})$ of scalar (vector-valued) functions, we denote by $L^{2}((0, T), Z)\left(\mathbf{L}^{2}((0, T), \mathbf{Z})\right)$ the Hilbert space and by $C([0, T] ; Z)$ $(\mathbf{C}([0, T], \mathbf{Z}))$ the Banach space of functions $v:[0, T] \rightarrow Z(\mathbf{v}:[0, T] \rightarrow \mathbf{Z})$. The spaces $H^{s}((0, T), Z), s \in \mathbb{R}_{+},\left(\mathbf{H}^{s}((0, T), \mathbf{Z})\right)$ are defined likewise.

We introduce the function spaces

$$
\begin{aligned}
\mathbf{V}(0, T) & :=\mathbf{H}^{1}\left((0, T), \mathbf{H}^{-1}(\Omega)\right) \cap \mathbf{L}^{2}\left((0, T), \mathbf{H}^{1}(\Omega)\right), \\
\mathbf{W}(0, T) & :=\left\{\mathbf{w} \in \mathbf{V}(0, T)|\mathbf{w}|_{\Sigma^{\prime}}=\mathbf{g},\left.\mathbf{w}\right|_{\Sigma_{\text {lat }}}=\mathbf{0}\right\}, \\
Q(0, T) & :=L^{2}\left((0, T), L_{0}^{2}(\Omega)\right),
\end{aligned}
$$

where $\Sigma^{\prime}=\Sigma_{\text {in }} \cup \Sigma_{\text {out }}$. Assuming $\left.\mathbf{F}(t) \in \mathbf{H}^{-1}(\Omega)\right), \mathbf{g}(t) \in \mathbf{H}_{00}^{1 / 2}\left(\Sigma^{\prime}\right), t \in(0, T)$, and $\mathbf{u}^{(0)} \in \mathbf{L}^{2}(\Omega)$, the weak formulation of the Navier-Stokes equations (2.1a)-(2.1e) requires the computation of $(\mathbf{u}, p) \in\left(\mathbf{W}(0, T) \cap \mathbf{L}^{\infty}(Q)\right) \times Q(0, T)$ such that for all 
$\mathbf{v} \in \mathbf{H}_{0}^{1}(\Omega)$ and $w \in L_{0}^{2}(\Omega)$ there holds

$$
\begin{aligned}
\left\langle\rho \frac{\partial \mathbf{u}}{\partial t}, \mathbf{v}\right\rangle+a(\mathbf{u}, \mathbf{v})-b(p, \mathbf{v}) & =\ell(\mathbf{v}), \\
b(w, \mathbf{u}) & =0 \\
\mathbf{u}(\cdot, 0) & =\mathbf{u}^{(0)} .
\end{aligned}
$$

Here, $a(\cdot, \cdot), b(\cdot, \cdot)$, and the functional $\ell(\cdot)$ are given by

$$
\begin{aligned}
a(\mathbf{u}, \mathbf{v}) & :=(\rho(\mathbf{u} \cdot \nabla) \mathbf{u}, \mathbf{v})_{0, \Omega}+(\nu \nabla \mathbf{u}, \nabla \mathbf{v})_{0, \Omega} \\
b(p, \mathbf{v}) & :=(p, \nabla \cdot \mathbf{v})_{0, \Omega} \quad, \quad \ell(v):=\langle\mathbf{F}, \mathbf{v}\rangle .
\end{aligned}
$$

We further suppose that $\Omega$ is filled with a suspension of $N$ viscoelastic particles immersed in the carrier fluid such that the subdomains $B_{t}^{(i)} \subset \Omega, 1 \leq i \leq N$, with $\bar{B}_{t}^{(i)} \cap \bar{B}_{t}^{(j)}=\emptyset, 1 \leq i \neq j \leq N$, describe the spatial location of the particles at time $t \in[0, T]$. The boundaries $\partial B_{t}^{(i)}$ are supposed to be non-selfintersecting closed curves. For ease of notation, we consider the case $N=1$ and write $B_{t}$ instead of $B_{t}^{(1)}$. The generalization to $N>1$ is obvious. We assume that the boundary $\partial B_{0}$ of the initial configuration $B_{0}$ has length $L:=\left|\partial B_{0}\right|$ and denote by $q \in[0, L]$ the Lagrangian coordinate labeling a material point on $\partial B_{0}$. We further refer to $\mathbf{X}(q, t)=\left(X_{1}(q, t), X_{2}(q, t)\right)^{T}$ as the position of that point at time $t \in(0, T]$. We denote by

$$
\mathcal{E}^{e}(\mathbf{X}(q, t))=\frac{\kappa_{e}}{2}\left|\frac{\partial \mathbf{X}}{\partial q}(q, t)\right|^{2}, \quad \mathcal{E}^{b}(\mathbf{X}(q, t))=\frac{\kappa_{b}}{2}\left|\frac{\partial^{2} \mathbf{X}}{\partial q^{2}}(q, t)\right|^{2},
$$

the local energy densities on the immersed elastic boundary $\partial B_{t}$, where $\kappa_{e}>0$ and $\kappa_{b}>0$ denotes the elasticity coefficient with respect to elongation-compression and bending, respectively. Then,

$$
\begin{gathered}
E(t):=E_{e}(t)+E_{b}(t), t \in(0, T), \\
E^{e}(t):=\int_{0}^{L} \mathcal{E}^{e}(\mathbf{X}(q, t)) d q, \quad E^{b}(t):=\int_{0}^{L} \mathcal{E}^{b}(\mathbf{X}(q, t)) d q,
\end{gathered}
$$

is the associated total energy consisting of the elastic energy $E^{e}(t)$ and the bending energy $E^{b}(t)$. The local force density $\mathbf{f}$ is given by $\mathbf{f}(q, t)=-E^{\prime}(\mathbf{X}(q, t))$, where $E^{\prime}$ stands for the the Gâteaux derivative of $E$.

We introduce the function space

$$
\mathbf{H}_{\text {per }}^{2}([0, L]):=\left\{\mathbf{Y} \in \mathbf{H}^{2}((0, L)) \mid \partial^{k} \mathbf{Y}(0) / \partial q^{k}=\partial^{k} \mathbf{Y}(L) / \partial q^{k}, k=0,1\right\},
$$

and we require

$$
\mathbf{X} \in \mathbf{H}^{1}\left((0, T), \mathbf{L}^{2}([0, L])\right) \cap \mathbf{L}^{2}\left((0, T), \mathbf{H}_{\text {per }}^{2}([0, L])\right),
$$

In view of $(2.4),(2.5)$ and $\mathbf{f}(\cdot, t)=-E^{\prime}(\mathbf{X}(\cdot, t))$ we have $\mathbf{f}(\cdot, t) \in \mathbf{H}_{\text {per }}^{2}([0, L])^{*}$. Then, assuming some initial configuration $\mathbf{X}^{(0)} \in \mathbf{H}_{\text {per }}^{2}([0, L])$, the variational formulation of the interaction equations reads

$$
\langle\mathbf{F}(t), \mathbf{v}\rangle=\langle\mathbf{f}(\cdot, t), \mathbf{v}(\mathbf{X}(\cdot, t))\rangle,
$$


for all $\mathbf{v} \in \mathbf{H}^{2+\mu}(\Omega) \cap \mathbf{H}_{0}^{1}(\Omega), \mu \geq 1 / 2$, such that $\left.\mathbf{v}\right|_{\partial B_{t}} \in \mathbf{H}_{\text {per }}^{2}([0, L])$, and

$$
\begin{aligned}
\int_{0}^{L} \frac{\partial \mathbf{X}}{\partial t}(q, t) \cdot \mathbf{Y}(q) d q & =\int_{0}^{L} \mathbf{u}(\mathbf{X}(q, t), t) \cdot \mathbf{Y}(q) d q, \\
\mathbf{X}(\cdot, 0) & =\mathbf{X}^{(0)}(\cdot),
\end{aligned}
$$

for all $\mathbf{Y} \in \mathbf{H}_{\mathrm{per}}^{2}([0, L])$.

REMARK 2.1. If we use (2.4) in (2.8a), for sufficiently smooth $\mathbf{v}$ we obtain

$$
\begin{aligned}
\langle\mathbf{F}(t), \mathbf{v}\rangle= & -\kappa_{e} \int_{0}^{L} \frac{\partial \mathbf{X}(\cdot, t)}{\partial q} \cdot \mathbf{D}^{1} \mathbf{v}(\mathbf{X}(\cdot, t)) \frac{\partial \mathbf{X}(\cdot, t)}{\partial q} d q \\
& -\kappa_{b} \int_{0}^{L} \frac{\partial^{2} \mathbf{X}(\cdot, t)}{\partial q^{2}} \cdot \mathbf{D}^{1} \mathbf{v}(\mathbf{X}(\cdot, t)) \frac{\partial^{2} \mathbf{X}(\cdot, t)}{\partial q^{2}} d q \\
& -\kappa_{b} \int_{0}^{L} \frac{\partial^{2} \mathbf{X}(\cdot, t)}{\partial q^{2}} \cdot \mathbf{D}^{2} \mathbf{v}(\mathbf{X}(\cdot, t))\left(\frac{\partial \mathbf{X}(\cdot, t)}{\partial q}, \frac{\partial \mathbf{X}(\cdot, t)}{\partial q}\right) d q .
\end{aligned}
$$

2.2. Fully implicit FE-IB. We assume $\mathcal{T}_{h}(\Omega)$ to be a simplicial triangulation of $\Omega$ that aligns with the partition of $\Gamma$. For $D \subseteq \bar{\Omega}$, we refer to $\mathcal{T}_{h}(D)$ as the union of triangles that have nonzero intersection with $D$, i.e.,

$$
\mathcal{T}_{h}(D)=\bigcup\left\{T \in \mathcal{T}_{h}(\Omega) \mid T \cap D \neq \emptyset\right\} .
$$

For $T \in \mathcal{T}_{h}(\Omega)$, we denote by $|T|$ the area of $T$ and by $h_{T}$ the diameter of $T$. We set $h:=\max \left\{h_{T} \mid T \in \mathcal{T}_{h}(\Omega)\right\}$. Further, $P_{k}(T), k \in \mathbb{N}$, refers to the set of polynomials of degree $\leq k$ on $T$. We suppose that $\mathcal{T}_{h}(\Omega)$ is quasi-uniform, i.e., there exist constants $0<c_{Q} \leq C_{Q}$ that only depend on the local geometry of the triangulation such that

$$
c_{Q} h \leq h_{T} \leq C_{Q} h, T \in \mathcal{T}_{h}(\Omega) .
$$

For the spatial discretization of the weak formulation (2.2a),(2.2b) of the incompressible Navier-Stokes equations we use P2-P1 Taylor-Hood elements [14], i.e., we define

$$
\begin{aligned}
V_{h} & :=\left\{v_{h} \in C(\bar{\Omega})\left|v_{h}\right|_{T} \in P_{2}(T), T \in \mathcal{T}_{h}(\Omega)\right\}, \\
\mathbf{V}_{h} & :=\left\{\mathbf{v}_{h} \in \mathbf{C}(\bar{\Omega}) \mid \mathbf{v}_{h}=\left(v_{h, 1}, v_{h, 2}\right)^{T}, v_{h, \nu} \in V_{h}\right\}, \\
Q_{h} & :=\left\{w_{h} \in C(\bar{\Omega})\left|w_{h}\right|_{T} \in P_{1}(T), T \in \mathcal{T}_{h}(\Omega)\right\} \cap L_{0}^{2}(\Omega),
\end{aligned}
$$

and set $\mathbf{V}_{h, 0}:=\mathbf{V}_{h} \cap \mathbf{C}_{0}(\bar{\Omega})$. The finite element spaces $V_{h}$ and $Q_{h}$ are spanned by the canonically specified nodal basis functions $\varphi_{h}^{(i)}, 1 \leq i \leq n_{1}$, and $\psi_{h}^{(i)}, 1 \leq i \leq N_{2}$. Assuming $\mathbf{g}_{h}$ to be the quadratic spline interpoland of $\mathbf{g}$ with respect to $\left.\mathcal{T}_{h}(\Omega)\right|_{\Gamma^{\prime}}$, $\Gamma^{\prime}=\Gamma_{\text {in }}$ resp. $\Gamma^{\prime}=\Gamma_{\text {out }}$, we set

$$
\begin{gathered}
\mathbf{W}_{h}(0, T):=\left\{\mathbf{w}_{h} \in \mathbf{C}([0, T], \mathbf{C}(\bar{\Omega}))\left|\mathbf{w}_{h}(\cdot, t)\right|_{T} \in \mathbf{V}_{h},\right. \\
\left.\left.\mathbf{w}_{h}(\cdot, t)\right|_{\Gamma^{\prime}}=\mathbf{g}_{h}(\cdot), t \in[0, T],\left.\mathbf{w}_{h}\right|_{\Sigma_{\text {lat }}}=\mathbf{0}\right\}, \\
Q_{h}(0, T):=\left\{w_{h} \in C([0, T] ; C(\bar{\Omega}))\left|w_{h}(\cdot, t)\right|_{T} \in Q_{h}, t \in[0, T]\right\} .
\end{gathered}
$$


The discretization of the immersed boundary is done with respect to a partition

$$
\mathcal{T}_{\Delta q}:=\left\{0=: q_{0}<q_{1}<\cdots<q_{n_{3}}:=L\right\}, n_{3} \in \mathbb{N},
$$

of the interval $[0, L]$ into subintervals $I_{i}:=\left[q_{i-1}, q_{i}\right], 1 \leq i \leq n_{3}$, of length $\Delta q_{i}:=$ $q_{i}-q_{i-1}$ with $\Delta q:=\max \left\{\Delta q_{i} \mid 1 \leq i \leq n_{3}\right\}$. We approximate $\mathbf{X}$ from (2.7) by periodic cubic splines and thus define

$$
\begin{aligned}
\mathbf{S}_{h}:=\left\{\mathbf{Y}_{h} \in \mathbf{C}^{2}([0, L] ; \Omega)\left|\mathbf{Y}_{h}\right|_{I_{i}} \in P_{3}\left(I_{i}\right)^{2},\right. \\
\\
\left.\quad 1 \leq i \leq m_{3}, \mathbf{Y}_{h}^{(k)}\left(q_{0}\right)=\mathbf{Y}_{h}^{(k)}\left(q_{M}\right), k=0,1,2\right\}
\end{aligned}
$$

where $P_{3}\left(I_{i}\right)$ stands for the set of polynomials of degree $\leq 3$ on $I_{i}$. By $\chi_{h}^{(i)}, 1 \leq i \leq n_{3}$, we denote the canonical basis functions (B-splines) spanning the scalar-valued counterpart of $\mathbf{S}_{h}$. The discrete immersed body occupies subdomains $B_{h, t} \subset \Omega, t \in[0, T]$, with boundaries $\partial B_{h, t}$ that are $C^{2}$ curves described by the periodic cubic spline $\mathbf{X}_{h}(\cdot, t) \in \mathbf{S}_{h}$.

We further consider a partitioning $\left\{0=: t_{0}<t_{1}<\cdots<t_{M}:=T\right\}$ of the time interval $[0, T]$ with step sizes $\tau_{k}:=t_{k+1}-t_{k}, 0 \leq k \leq M-1$, and approximate the time derivatives $\partial \mathbf{u}_{h} / \partial t$ and $\partial \mathbf{X}_{h} / \partial t$ at $t_{k+1}$ by the backward difference quotient. Denoting by $\mathbf{u}_{h}^{(k)} \in \mathbf{V}_{h}, p_{h}^{(k)} \in Q_{h}$, and $\mathbf{X}_{h}^{(k)} \in \mathbf{S}_{h}, 0 \leq k \leq M$, approximations of $\mathbf{u}\left(\cdot, t_{k}\right), p\left(\cdot, t_{k}\right)$, and $\mathbf{X}\left(\cdot, t_{k}\right)$, the fully implicit FE-IB method (BE/BE FE-IB) amounts to the computation of $\left(\mathbf{u}_{h}^{(k+1)}, p_{h}^{(k+1)}, \mathbf{X}_{h}^{(k+1)}\right), 0 \leq k \leq M-1$, such that for all $\mathbf{v}_{h} \in \mathbf{V}_{h, 0}, w_{h} \in Q_{h}$, and all $\mathbf{Y}_{h} \in \mathbf{S}_{h}$ there holds

$$
\begin{aligned}
\rho\left(\mathbf{u}_{h}^{(k+1)}, \mathbf{v}_{h}\right)_{0, \Omega}+\tau_{k} a\left(\mathbf{u}_{h}^{(k+1)}, \mathbf{v}_{h}\right)-\tau_{k} b\left(p_{h}^{(k+1)}, \mathbf{v}_{h}\right) & =\ell_{h}\left(\mathbf{v}_{h}\right) \\
b\left(w_{h}, \mathbf{u}_{h}^{(k+1)}\right) & =0 \\
\int_{0}^{L} \mathbf{X}_{h}^{(k+1)} \cdot \mathbf{Y}_{h}(q) d q-\tau_{k} \int_{0}^{L} \mathbf{u}_{h}\left(\mathbf{X}_{h}^{(k+1)}\right) \cdot \mathbf{Y}_{h} d q & =\int_{0}^{L} \mathbf{X}_{h}^{(k)} \cdot \mathbf{Y}_{h} d q
\end{aligned}
$$

where the right-hand side in $(2.12 \mathrm{a})$ is given by

$$
\begin{aligned}
& \ell_{h}\left(\mathbf{v}_{h}\right):=\rho\left(\mathbf{u}_{h}^{(k)}, \mathbf{v}_{h}\right)_{0, \Omega}+\tau_{k} \int_{0}^{L} \mathbf{f}_{h}^{(k+1)}(q) \cdot \mathbf{v}_{h}\left(\mathbf{X}_{h}^{(k+1)}\right) d q \\
& \int_{0}^{L} \mathbf{f}_{h}^{(k+1)}(q) \cdot \mathbf{v}_{h}\left(\mathbf{X}_{h}^{(k+1)}\right) d q:=-\kappa_{e} \int_{0}^{L} \frac{\partial \mathbf{X}_{h}^{(k+1)}}{\partial q} \cdot \mathbf{D}^{1} \mathbf{v}_{h}\left(\mathbf{X}_{h}^{(k+1)}\right) \frac{\partial \mathbf{X}_{h}^{(k+1)}}{\partial q} d q \\
& -\kappa_{b} \int_{0}^{L} \frac{\partial^{2} \mathbf{X}_{h}^{(k+1)}}{\partial q^{2}} \cdot \mathbf{D}^{1} \mathbf{v}_{h}\left(\mathbf{X}_{h}^{(k+1)}\right) \frac{\partial^{2} \mathbf{X}_{h}^{(k+1)}}{\partial q^{2}} d q \\
& -\kappa_{b} \int_{0}^{L} \frac{\partial^{2} \mathbf{X}_{h}^{(k+1)}}{\partial q^{2}} \cdot \mathbf{D}^{2} \mathbf{v}_{h}\left(\mathbf{X}_{h}^{(k+1)}\right)\left(\frac{\partial \mathbf{X}_{h}^{(k+1)}}{\partial q}, \frac{\partial \mathbf{X}_{h}^{(k+1)}}{\partial q}\right) d q .
\end{aligned}
$$

REMARK 2.2. We note that $\mathbf{v}_{h} \in \mathbf{V}_{h, 0}$ does not satisfy $\left.\mathbf{v}_{h}\right|_{\partial B_{h, t_{k+1}}} \in \mathbf{H}_{\mathrm{per}}^{2}([0, L])$ (cf. (2.8a)). However, assuming meas $\left(B_{h, t_{k+1}} \cap \mathcal{E}_{h}(\Omega)\right)=\emptyset$, it follows that (2.13) is well defined for $\mathbf{v}_{h} \in \mathbf{V}_{h, 0}$ and $\mathbf{X}_{h}^{(k+1)} \in \mathbf{S}_{h}$. 
For the algebraic formulation of the BE/BE FE-IB (2.12a)-(2.12c), we introduce the vectors

$$
\begin{aligned}
\mathbf{u}^{(k)} & :=\left(u_{1}^{(k)}, \cdots, u_{n_{1}}^{(k)}, u_{n_{1}+1}^{(k)}, \cdots, u_{N_{1}}^{(k)}\right)^{T}, 0 \leq k \leq M, \\
\mathbf{p}^{(k)} & :=\left(p_{1}^{(k)}, \cdots, p_{N_{2}}^{(k)}\right)^{T}, 0 \leq k \leq M, \\
\mathbf{X}^{(k)} & :=\left(X_{1}^{(k)}, \cdots, X_{n_{3}}^{(k)}, X_{n_{3}+1}^{(k)}, \cdots, X_{N_{3}}^{(k)}\right)^{T}, 0 \leq k \leq M,
\end{aligned}
$$

the mass matrices $\mathbf{M}_{\nu} \in \mathbb{R}^{N_{\nu} \times N_{\nu}}, \mathbf{M}_{\nu}=\operatorname{blockdiag}\left(\mathbf{M}_{\nu}^{(1)}, \mathbf{M}_{\nu}^{(2)}\right), \nu \in\{1,3\}$, the stiffness matrix $\mathbf{A} \in \mathbb{R}^{N_{1} \times N_{1}}, \mathbf{A}=\operatorname{blockdiag}\left(\mathbf{A}^{(1)}, \mathbf{A}^{(2)}\right)$, as well as the matrix $\mathbf{B} \in \mathbb{R}^{N_{2} \times N_{1}}$ by means of

$$
\begin{aligned}
\left(\mathbf{M}_{1}^{(\mu)}\right)_{i j} & :=\rho \int_{\Omega} \varphi_{h}^{(i)} \varphi_{h}^{(j)} d \mathbf{x}, 1 \leq i, j \leq n_{1}, 1 \leq \mu \leq 2, \\
\left(\mathbf{M}_{3}^{(\mu)}\right)_{i j} & :=\rho \int_{0}^{L} \chi_{h}^{(i)} \chi_{h}^{(j)} d q, 1 \leq i, j \leq n_{3}, 1 \leq \mu \leq 2, \\
\mathbf{A}_{i j}^{(\nu)} & :=\nu \int_{\Omega} \nabla \varphi_{h}^{(i)} \cdot \nabla \varphi_{h}^{(j)} d \mathbf{x}, 1 \leq i, j \leq n_{1}, 1 \leq \nu \leq 2, \\
\mathbf{B}_{i j} & :=\int_{\Omega} \frac{\partial \varphi_{h}^{(j)}}{\partial x_{1}} \psi_{h}^{(i)} d \mathbf{x}, \mathbf{B}_{i, n_{1}+j}:=\int_{\Omega} \frac{\partial \varphi_{h}^{(j)}}{\partial x_{2}} \psi_{h}^{(i)} d \mathbf{x}, 1 \leq i \leq N_{2}, 1 \leq j \leq n_{1} .
\end{aligned}
$$

We note that the matrices $\mathbf{M}_{1}, \mathbf{M}_{3}$, and $\mathbf{A}$ are symmetric and positive definite. In particular, there exist constants $\mu_{1}>0, \mu_{3}>0$, and $\alpha>0$ such that for $\mathbf{v} \in \mathbb{R}^{N_{1}}$ and $\mathbf{Y} \in \mathbb{R}^{N_{3}}$ there holds

$$
\mathbf{v}^{T} \mathbf{M}_{1} \mathbf{v} \geq \mu_{1}\|\mathbf{v}\|^{2}, \quad \mathbf{Y}^{T} \mathbf{M}_{3} \mathbf{Y} \geq \mu_{3}\|\mathbf{Y}\|^{2}, \quad \mathbf{v}^{T} \mathbf{A} \mathbf{v} \geq \alpha\|\mathbf{v}\|^{2}
$$

The matrix $\mathbf{B}^{T}$ satisfies an inf-sup condition, or equivalently

$$
\left\|\mathbf{B}^{T} \mathbf{q}\right\|^{2} \geq \beta\|\mathbf{q}\|_{\mathbb{R}^{N_{2}} \backslash \mathbb{R}^{1}}^{2}, \quad \beta>0, \mathbf{q} \in \mathbb{R}^{N_{2}} \backslash \operatorname{span}\left\{(1, \ldots, 1)^{T}\right\} .
$$

We further define the nonlinear mappings $\mathbf{C}: \mathbb{R}^{N_{1}} \rightarrow \mathbb{R}^{N_{1}}, \mathbf{F}: \mathbb{R}^{N_{3}} \rightarrow \mathbb{R}^{N_{1}}$, and $\mathbf{K}: \mathbb{R}^{N_{3}} \rightarrow \mathbb{R}^{N_{3} \times N_{1}}$ according to

$$
\begin{aligned}
& (\mathbf{C}(\mathbf{u}))_{(\ell-1) n_{1}+i}:=\sum_{j, k=1}^{n_{1}} \sum_{m=1}^{2} \rho \int_{\Omega} \varphi_{h}^{(i)} \varphi_{h}^{(j)} \frac{\partial \varphi_{h}^{(k)}}{\partial x_{m}} d \mathbf{x} u_{(m-1) n_{1}+j} u_{(\ell-1) n_{1}+k} \\
& \left(\mathbf{F}\left(\mathbf{X}^{(k+1)}\right)\right)_{(\ell-1) n_{1}+i}:=\int_{0}^{L} \mathbf{f}_{h, \ell}^{(k+1)}(q) \varphi_{h}^{(i)}\left(\mathbf{X}_{h}^{(k+1)}\right) d q
\end{aligned}
$$

where $1 \leq \ell \leq 2,1 \leq i \leq n_{1}$, and

$$
\left(\mathbf{K}\left(\mathbf{X}^{(k+1)}\right)\right)_{(\ell-1) n_{3}+i,(\ell-1) n_{1}+j}:=\int_{0}^{L} \varphi_{h}^{(j)}\left(\mathbf{X}_{h}^{(k+1)}\right) \chi_{h}^{(i)} d q
$$


(and zero else), where $1 \leq \ell \leq 2,1 \leq i \leq n_{3}, 1 \leq j \leq n_{1}$, and $\mathbf{X}_{h}^{(k+1)}:=\left(\sum_{i=1}^{n_{3}} X_{i} \chi_{h}^{(i)}\right.$, $\left.\sum_{i=1}^{n_{3}} X_{n_{3}+i} \chi_{h}^{(i)}\right)^{T}$

For $\mathbf{z}=(\mathbf{u}, \mathbf{p}, \mathbf{X})^{T} \in \mathbb{R}^{N_{1}+N_{2}+N_{3}}$ let us define the nonlinear function $\mathbf{H}: \mathbb{R}^{N} \rightarrow \mathbb{R}^{N}$, $N:=N_{1}+N_{2}+N_{3}$, by

$$
\mathbf{H}(\mathbf{z}):=-\left(\begin{array}{l}
(\mathbf{A}+\mathbf{C}(\mathbf{u})) \mathbf{u}+\mathbf{B}^{T} \mathbf{p}-\mathbf{F}(\mathbf{X})-\mathbf{F}_{0} \\
\mathbf{B} \mathbf{u}-\mathbf{b} \\
-\mathbf{K}(\mathbf{X}) \mathbf{u}
\end{array}\right) \in \mathbb{R}^{N}
$$

Note that the first two lines in (2.16) describe the finite element discretized stationary incompressible Navier-Stokes equations and that the vectors $\mathbf{F}_{0} \in \mathbb{R}^{N_{1}}$ and $\mathbf{b} \in \mathbb{R}^{N_{2}}$ result from the inhomogeneous boundary data on $\Sigma_{\text {in }}$ and $\Sigma_{\text {out }}$. We remind that the third line in (2.16) stems from the variational form of the equations of motion for the immersed boundary.

Denoting by $\mathbf{M}=\operatorname{blockdiag}\left(\mathbf{M}_{1}, \mathbf{0}, \mathbf{M}_{3}\right) \in \mathbb{R}^{N \times N}$ the (singular) mass matrix associated with $\left(\mathbf{u}_{h}, \mathbf{0}, \mathbf{X}_{h}\right)$, the ODE describing the dynamics of the immersed boundaryfluid interaction can be written as

$$
\begin{aligned}
\mathbf{M} \dot{\mathbf{z}}(t) & =\mathbf{H}(\mathbf{z}(t)), t \in[0, T] \\
\mathbf{z}(0) & =\mathbf{z}_{0}
\end{aligned}
$$

which is equivalent to the formulation as a Volterra equation of the second kind

$$
\mathbf{0}=\mathbf{M} \mathbf{z}(t)-\mathbf{M} \mathbf{z}_{0}-\int_{0}^{t} \mathbf{H}(\mathbf{z}(s)) d s=: \widetilde{\mathbf{G}}(\mathbf{z}(t) ; t) .
$$

This represents a parameter-dependent nonlinear system of equations with trajectory $\{\mathbf{z}(t) \mid t \in[0, T]\}$. The numerical solution by means of Newton's method is not suitable, since it would require some global information of the Jacobian $\mathbf{H}^{\prime}(\mathbf{z}(\cdot))$ up to the point $\mathbf{z}(t)$ and, in addition, an exact evaluation of the integral in (2.17).

To overcome these difficulties, we approximate (2.17) by the backward Euler (BE) scheme

$$
\mathbf{0}=\mathbf{M} \mathbf{z}\left(t_{k+1}\right)-\mathbf{M} \mathbf{z}_{k}-\tau_{k} \mathbf{H}\left(\mathbf{z}\left(t_{k+1}\right)\right), k \geq 0,
$$

where $\tau_{k}:=t_{k+1}-t_{k}$, i.e., for each time step we have to compute the root $\mathbf{z}\left(t_{k+1}\right)$ of the nonlinear function

$$
\mathbf{G}\left(\mathbf{z} ; t_{k}+\tau_{k}\right):=\mathbf{M} \mathbf{z}-\mathbf{M} \mathbf{z}\left(t_{k}\right)-\tau_{k} \mathbf{H}(\mathbf{z})
$$

Consequently, the problem we deal with is a time-discretized approximation of a parameter-dependent nonlinear system of equations, and thus can be solved by the methodology presented in section 3 .

Setting $\mathbf{z}^{(k)}:=\left(\mathbf{u}^{(k)}, \mathbf{p}^{(k)}, \mathbf{X}^{(k)}\right)^{T}, 0 \leq k \leq M$, at each time step the BE/BE FE-IB then amounts to the computation of $\mathbf{z}^{(k+1)}, 0 \leq k \leq M-1$, as the solution of the nonlinear system

$$
\mathbf{G}\left(\mathbf{z}^{(k+1)} ; t_{k+1}\right)=\mathbf{0}
$$


where the nonlinear mapping $\mathbf{G}\left(\cdot ; t_{k+1}\right): \mathbb{R}^{N} \rightarrow \mathbb{R}^{N}$ relative to $\left(\tau_{k}, \mathbf{z}\left(t_{k}\right)\right)$ is given by

$$
\begin{aligned}
& \mathbf{G}\left(\mathbf{z} ; t_{k+1}\right):= \\
& \left(\begin{array}{c}
\left(\mathbf{M}_{1}+\tau_{k} \mathbf{A}\right) \mathbf{u}+\tau_{k} \mathbf{C}(\mathbf{u})+\tau_{k} \mathbf{B}^{T} \mathbf{p}-\tau_{k} \mathbf{F}(\mathbf{X})-\mathbf{M}_{1} \mathbf{u}^{(k)}-\tau_{k} \mathbf{F}_{0} \\
\mathbf{B u}-\mathbf{b} \\
\mathbf{M}_{3} \mathbf{X}-\mathbf{M}_{3} \mathbf{X}^{(k)}-\tau_{k} \mathbf{K}(\mathbf{X}) \mathbf{u}
\end{array}\right),
\end{aligned}
$$

where for scaling reasons we have multiplied the second block row by $1 / \tau_{k}$. The Jacobian $\mathbf{G}^{\prime}\left(\mathbf{z} ; t_{k+1}\right) \in \mathbb{R}^{N \times N}$ reads as follows:

$$
\mathbf{G}^{\prime}\left(\mathbf{z} ; t_{k+1}\right):=\left(\begin{array}{ccc}
\mathbf{M}_{1}+\tau_{k} \mathbf{A}+\tau_{k} \mathbf{C}^{\prime}(\mathbf{u}) & \tau_{k} \mathbf{B}^{T} & -\tau_{k} \mathbf{F}^{\prime}(\mathbf{X}) \\
\mathbf{B} & \mathbf{0} & \mathbf{0} \\
-\tau_{k} \mathbf{K}(\mathbf{X}) & \mathbf{0} & \mathbf{M}_{3}-\tau_{k} \mathbf{K}_{\mathbf{X}}^{\prime}(\mathbf{X}, \mathbf{u})
\end{array}\right) .
$$

Here, $\mathbf{C}^{\prime}(\mathbf{u}) \in \mathbb{R}^{N_{1} \times N_{1}}$ and $\mathbf{F}^{\prime}(\mathbf{X}) \in \mathbb{R}^{N_{1} \times N_{3}}$ are the Fréchet derivatives of $\mathbf{C}$ and $\mathbf{F}$ at $\mathbf{u}$ and $\mathbf{X}$, respectively, whereas $\mathbf{K}_{\mathbf{X}}^{\prime}(\mathbf{X}, \mathbf{u}) \in \mathbb{R}^{N_{3} \times N_{3}}$ stands for the partial Fréchet derivative of $\mathbf{K}(\mathbf{X}) \mathbf{u}$ with respect to $\mathbf{X}$ at $(\mathbf{X}, \mathbf{u})$.

The solution of the BE/BE FE-IB by a predictor-corrector continuation strategy in time requires the invertibility of the Jacobian which can be guaranteed under some restriction of the time step size.

TheOREM 2.3. For a given $\mathbf{z} \in \mathbb{R}^{N}$ and $\tau^{\min }>0$, assume that the time step size $\tau_{k}$ satisfies

$$
\begin{aligned}
& 0<\tau^{\min } \leq \tau_{k} \leq \tau_{k}^{\max }(\mathbf{z}):=\min \left(\lambda_{1}, \lambda_{2}\right), \\
& \lambda_{1}:=-\frac{c_{1}}{c_{2}}+\sqrt{\frac{\mu_{1}}{2 c_{2}}+\left(\frac{c_{1}}{c_{2}}\right)^{2}}, \quad \lambda_{2}:=-\frac{d_{1}}{d_{2}}+\sqrt{\frac{\mu_{3}}{2 d_{2}}+\left(\frac{d_{1}}{d_{2}}\right)^{2}}
\end{aligned}
$$

where $c_{\nu}, d_{\nu}, 1 \leq \nu \leq 2$, depend on $\mathbf{z}$ and are given by

$$
\begin{aligned}
c_{1} & :=\left\|\mathbf{C}^{\prime}(\mathbf{u})\right\|+\frac{1}{2}\left\|\mathbf{F}^{\prime}(\mathbf{X})\right\|+\frac{1}{4}\|\mathbf{K}(\mathbf{X})\|^{2} /\left\|\mathbf{M}_{1}\right\|^{2}-\alpha, \\
c_{2} & :=3 \mu_{1}\left(\|\mathbf{A}\|^{2}+\left\|\mathbf{C}^{\prime}(\mathbf{u})\right\|^{2}\right), \\
d_{1} & :=\frac{1}{2}\left(\left\|\mathbf{M}_{1}\right\|^{2}\left\|\mathbf{F}^{\prime}(\mathbf{X})\right\|+\frac{1}{2}\|\mathbf{K}(\mathbf{X})\|+\left\|\mathbf{K}_{\mathbf{X}}^{\prime}(\mathbf{X}, \mathbf{u})\right\|\right), \\
d_{2} & :=3 \mu_{1}\left\|\mathbf{F}^{\prime}(\mathbf{X})\right\|^{2} .
\end{aligned}
$$

Then, the Jacobian $\mathbf{G}^{\prime}\left(\mathbf{z} ; t_{k+1}\right) \in \mathbb{R}^{N \times N}$ is invertible. In particular, there holds

$$
\begin{aligned}
& \left\|\left(\mathbf{G}^{\prime}\left(\mathbf{z} ; t_{k+1}\right)\right)^{-1}\right\| \leq \gamma^{-1}, \quad \gamma=\gamma(\mathbf{z}):=\frac{\kappa_{1}}{\kappa_{2}}, \\
& \kappa_{1}:=\frac{1}{2} \min \left(\mu_{1}\left\|\mathbf{M}_{1}\right\|^{2}, \frac{3}{4}\left(\tau^{\min }\right)^{2} \beta \mu_{1}, \mu_{3}\right), \\
& \kappa_{2}:=\left(\max \left(8\left\|\mathbf{M}_{1}\right\|^{4}+2\|\mathbf{B}\|^{2},\left(\tau_{k}^{\max }\right)^{2}\left(8\left\|\mathbf{M}_{1}\right\|^{4}+\frac{\mu_{1}^{2}}{2}\left\|\mathbf{B}^{T}\right\|^{2}\right), 1\right)\right)^{1 / 2} .
\end{aligned}
$$

Proof. For $\mathbf{w}=(\mathbf{v}, \mathbf{q}, \mathbf{Y})^{T}$ we choose $\overline{\mathbf{w}}=(\overline{\mathbf{v}}, \overline{\mathbf{q}}, \overline{\mathbf{Y}})^{T}$ according to $\overline{\mathbf{v}}=2\left\|\mathbf{M}_{1}\right\|^{2} \mathbf{v}+$ 
$\mu_{1} \tau_{k} \mathbf{B}^{T} \mathbf{q} / 2, \overline{\mathbf{q}}=\mathbf{B v}-2 \tau_{k}\left\|\mathbf{M}_{1}\right\|^{2} \mathbf{q}$, and $\overline{\mathbf{Y}}=\mathbf{Y}$. It follows that

$$
\begin{aligned}
& \overline{\mathbf{w}}^{T} G^{\prime}\left(\mathbf{z} ; t_{k+1}\right) \mathbf{w}= \\
& \left(2\left\|\mathbf{M}_{1}\right\|^{2} \mathbf{v}+\frac{1}{2} \mu_{1} \tau_{k} \mathbf{B}^{T} \mathbf{q}\right)^{T}\left(\mathbf{M}_{1}+\tau_{k} \mathbf{A}+\tau_{k} \mathbf{C}^{\prime}(\mathbf{u})\right) \mathbf{v} \\
& +\tau_{k}\left(2\left\|\mathbf{M}_{1}\right\|^{2} \mathbf{v}+\frac{1}{2} \mu_{1} \tau_{k} \mathbf{B}^{T} \mathbf{q}\right)^{T} \mathbf{B}^{T} \mathbf{q} \\
& -\tau_{k}\left(2\left\|\mathbf{M}_{1}\right\|^{2} \mathbf{v}+\frac{1}{2} \mu_{1} \tau_{k} \mathbf{B}^{T} \mathbf{q}\right)^{T} \mathbf{F}^{\prime}(\mathbf{X}) \mathbf{Y} \\
& +\left(\mathbf{B v}-2 \tau_{k}\left\|\mathbf{M}_{1}\right\|^{2} \mathbf{q}\right)^{T} \mathbf{B} \mathbf{v} \\
& -\tau_{k} \mathbf{Y}^{T} \mathbf{K}(\mathbf{X}) \mathbf{v}+\mathbf{Y}^{T}\left(\mathbf{M}_{3}-\tau_{k} \mathbf{K}_{\mathbf{X}}^{\prime}(\mathbf{X}, \mathbf{u})\right) \mathbf{Y}
\end{aligned}
$$

For the first term on the right-hand side in (2.25) we obtain

$$
\begin{aligned}
& \left(2\left\|\mathbf{M}_{1}\right\|^{2} \mathbf{v}+\frac{1}{2} \mu_{1} \tau_{k} \mathbf{B}^{T} \mathbf{q}\right)^{T}\left(\mathbf{M}_{1}+\tau_{k} \mathbf{A}+\tau_{k} \mathbf{C}^{\prime}(\mathbf{u})\right) \mathbf{v}= \\
& 2\left\|\mathbf{M}_{1}\right\|^{2} \mathbf{v}^{T}\left(\mathbf{M}_{1}+\tau_{k} \mathbf{A}+\tau_{k} \mathbf{C}^{\prime}(\mathbf{u})\right) \mathbf{v}+\frac{1}{2} \mu_{1} \tau_{k}\left(\mathbf{B}^{T} \mathbf{q}\right)^{T}\left(\mathbf{M}_{1}+\tau_{k} \mathbf{A}+\tau_{k} \mathbf{C}^{\prime}(\mathbf{u})\right) \mathbf{v} .
\end{aligned}
$$

Using (2.14), the first term on the right-hand side in (2.26) can be estimated from below as follows

$$
\begin{aligned}
& 2\left\|\mathbf{M}_{1}\right\|^{2} \mathbf{v}^{T}\left(\mathbf{M}_{1}+\tau_{k} \mathbf{A}+\tau_{k} \mathbf{C}^{\prime}(\mathbf{u})\right) \mathbf{v} \geq \\
& 2\left\|\mathbf{M}_{1}\right\|^{2} \underbrace{\mathbf{v}^{T} \mathbf{M}_{1} \mathbf{v}}_{\geq \mu_{1}\|\mathbf{v}\|^{2}}+2 \tau_{k}\left\|\mathbf{M}_{1}\right\|^{2} \underbrace{\mathbf{v}^{T} \mathbf{A v}}_{\geq \alpha\|\mathbf{v}\|^{2}}-2 \tau_{k}\left\|\mathbf{M}_{1}\right\|^{2}\left\|\mathbf{C}^{\prime}(\mathbf{u})\right\|\|\mathbf{v}\|^{2} .
\end{aligned}
$$

Using Young's inequality, the second term on the right-hand side in (2.26) can be estimated from above according to

$$
\begin{aligned}
& \frac{1}{2} \mu_{1} \tau_{k}\left(\mathbf{B}^{T} \mathbf{q}\right)^{T}\left(\mathbf{M}_{1}+\tau_{k} \mathbf{A}+\tau_{k} \mathbf{C}^{\prime}(\mathbf{u})\right) \mathbf{v} \leq \\
& \frac{\sqrt{\mu_{1}}}{\sqrt{2}}\left\|\mathbf{M}_{1}\right\|\|\mathbf{v}\| \frac{\sqrt{\mu_{1}}}{\sqrt{2}} \tau_{k}\left\|\mathbf{B}^{T} \mathbf{q}\right\|+\tau_{k}^{2} \frac{\mu_{1}}{2}\|\mathbf{A}\|\|\mathbf{v}\|\left\|\mathbf{B}^{T} \mathbf{q}\right\|+\tau_{k}^{2} \frac{\mu_{1}}{2}\left\|\mathbf{C}^{\prime}(\mathbf{u})\right\|\|\mathbf{v}\|\left\|\mathbf{B}^{T} \mathbf{q}\right\| \leq \\
& \frac{1}{8} \frac{\mu_{1}}{2} \tau_{k}^{2}\left\|\mathbf{B}^{T} \mathbf{q}\right\|^{2}+2 \frac{\mu_{1}}{2}\left\|\mathbf{M}_{1}\right\|^{2}\|\mathbf{v}\|^{2}+\frac{1}{24} \frac{\mu_{1}}{2} \tau_{k}^{2}\left\|\mathbf{B}^{T} \mathbf{q}\right\|^{2}+6 \frac{\mu_{1}}{2} \tau_{k}^{2}\|\mathbf{A}\|^{2}\|\mathbf{v}\|^{2} \\
& +\frac{1}{24} \frac{\mu_{1}}{2} \tau_{k}^{2}\left\|\mathbf{B}^{T} \mathbf{q}\right\|^{2}+6 \frac{\mu_{1}}{2} \tau_{k}^{2}\left\|\mathbf{C}^{\prime}(\mathbf{u})\right\|^{2}\|\mathbf{v}\|^{2}
\end{aligned}
$$

For the second term on the right-hand side in (2.25) it follows that

$$
\tau_{k}\left(2\left\|\mathbf{M}_{1}\right\|^{2} \mathbf{v}+\frac{1}{2} \mu_{1} \tau_{k} \mathbf{B}^{T} \mathbf{q}\right)^{T} \mathbf{B}^{T} \mathbf{q}=2 \tau_{k}\left\|\mathbf{M}_{1}\right\|^{2} \mathbf{v}^{T} \mathbf{B}^{T} \mathbf{q}+\frac{\mu_{1}}{2} \tau_{k}^{2}\left\|\mathbf{B}^{T} \mathbf{q}\right\|^{2} .
$$

Another application of Young's inequality reveals that the third term on the righthand side in (2.26) can be estimated from above by means of

$$
\begin{aligned}
& \tau_{k}\left(2\left\|\mathbf{M}_{1}\right\|^{2} \mathbf{v}+\frac{1}{2} \mu_{1} \tau_{k} \mathbf{B}^{T} \mathbf{q}\right)^{T} \mathbf{F}^{\prime}(\mathbf{X}) \mathbf{Y}= \\
& 2 \tau_{k}\left\|\mathbf{M}_{1}\right\|^{2} \mathbf{v}^{T} \mathbf{F}^{\prime}(\mathbf{X}) \mathbf{Y}+\frac{\mu_{1}}{2} \tau_{k}^{2}\left(\mathbf{B}^{T} \mathbf{q}\right)^{T} \mathbf{F}^{\prime}(\mathbf{X}) \mathbf{Y} \leq \\
& \tau_{k}\left\|\mathbf{M}_{1}\right\|^{2}\left\|\mathbf{F}^{\prime}(\mathbf{X})\right\|\left(\|\mathbf{v}\|^{2}+\|\mathbf{Y}\|^{2}\right)+\frac{1}{24} \frac{\mu_{1}}{2} \tau_{k}^{2}\left\|\mathbf{B}^{T} \mathbf{q}\right\|^{2}+6 \frac{\mu_{1}}{2} \tau_{k}^{2}\left\|\mathbf{F}^{\prime}(\mathbf{X})\right\|^{2}\|\mathbf{Y}\|^{2}
\end{aligned}
$$


For the fourth term on the right-hand side in (2.26) we obtain

$$
\left(\mathbf{B v}-2 \tau_{k}\left\|\mathbf{M}_{1}\right\|^{2} \mathbf{q}\right)^{T} \mathbf{B v}=\underbrace{\|\mathbf{B} \mathbf{v}\|^{2}}_{\geq 0}-2 \tau_{k}\left\|\mathbf{M}_{1}\right\|^{2} \mathbf{q}^{T} \mathbf{B v} .
$$

Using Young's inequality again, the fifth term on the right-hand side in (2.26) can be estimated from above as follows

$$
\tau_{k} \mathbf{Y}^{T} \mathbf{K}(\mathbf{X}) \mathbf{v} \leq \frac{1}{2} \tau_{k}\|\mathbf{K}(\mathbf{X})\|\left(\|\mathbf{v}\|^{2}+\|\mathbf{Y}\|^{2}\right) .
$$

Finally, in view of $(2.14)$, for the sixth term on the right-hand side in (2.26) we get the lower bound

$$
\left(\mathbf{M}_{3}-\tau_{k} \mathbf{K}_{\mathbf{X}}^{\prime}(\mathbf{X}, \mathbf{u})\right) \mathbf{Y} \geq \underbrace{\mathbf{Y}^{T} \mathbf{M}_{3} \mathbf{Y}}_{\geq \mu_{3}\|\mathbf{Y}\|^{2}}-\tau_{k}\left\|\mathbf{K}_{\mathbf{X}}^{\prime}(\mathbf{X}, \mathbf{u})\right\|\|\mathbf{Y}\|^{2}
$$

Summarizing (2.26)-(2.33), from (2.25) we deduce

$$
\begin{aligned}
& \overline{\mathbf{w}}^{T} G^{\prime}\left(\mathbf{z} ; t_{k+1}\right) \mathbf{w} \geq \\
& \left\|\mathbf{M}_{1}\right\|^{2}\left(\mu_{1}-2 c_{1} \tau_{k}-c_{2} \tau_{k}^{2}\right)\|\mathbf{v}\|^{2}+\frac{3}{8} \tau_{k}^{2} \mu_{1}\left\|\mathbf{B}^{T} \mathbf{q}\right\|^{2}+\left(\mu_{3}-2 d_{1} \tau_{k}-d_{2} \tau_{k}^{2}\right)\|\mathbf{Y}\|^{2} .
\end{aligned}
$$

We choose $\tau_{k}$ such that

$$
\mu_{1}-2 c_{1} \tau_{k}-c_{2} \tau_{k}^{2} \geq \frac{1}{2} \mu_{1}, \quad \mu_{3}-2 d_{1} \tau_{k}-d_{2} \tau_{k}^{2} \geq \frac{1}{2} \mu_{3},
$$

which is satisfied, if $\tau_{k}$ obeys the upper bound in (2.22). On the other hand, using (2.15) and the lower bound in (2.22), it follows that

$$
\frac{3}{8} \tau_{k}^{2} \mu_{1}\left\|\mathbf{B}^{T} \mathbf{q}\right\|^{2} \geq \frac{3}{8}\left(\tau_{k}^{(\min )}\right)^{2} \beta \mu_{1}\|\mathbf{q}\|_{\mathbb{R}^{N_{2}} \backslash \mathbb{R}^{1}}^{2}
$$

Taking advantage of (2.35) and (2.36) in (2.34), we finally obtain

$$
\overline{\mathbf{w}}^{T} G^{\prime}\left(\mathbf{z} ; t_{k+1}\right) \mathbf{w} \geq \kappa_{1}\|\mathbf{w}\|^{2} .
$$

Consequently, in view of

$$
\|\overline{\mathbf{w}}\| \leq \kappa_{2}\|\mathbf{w}\|
$$

$\mathbf{G}^{\prime}\left(\mathbf{z} ; t_{k+1}\right)$ satisfies the inf-sup condition

$$
\inf _{\mathbf{w} \neq 0} \sup _{\hat{\mathbf{w}} \neq 0} \frac{\hat{\mathbf{w}}^{T} \mathbf{G}^{\prime}\left(\mathbf{z} ; t_{k+1}\right) \mathbf{w}}{\|\mathbf{w}\|\|\hat{\mathbf{w}}\|} \geq \inf _{\mathbf{w} \neq 0} \frac{\overline{\mathbf{w}}^{T} \mathbf{G}^{\prime}\left(\mathbf{z} ; t_{k+1}\right) \mathbf{w}}{\|\mathbf{w}\|\|\overline{\mathbf{w}}\|} \geq \gamma,
$$

from which we deduce bijectivity by means of the generalized Lax-Milgram lemma (cf., e.g., $[12,13])$.

REMARK 2.4. We may expect a time step restriction, if the convective term and/or the deformation of the particle is dominant, i.e., in case $\left\|\mathbf{C}^{\prime}(\mathbf{u})\right\|$ and/or $\left\|\mathbf{F}^{\prime}(\mathbf{X})\right\|$ is large. These scenarios are reflected by the time step restriction (2.22). Usually, the step size restriction (2.22) is relatively mild and not the reason why we use an adaptive continuation strategy. The adaptivity is due to the convergence requirements of the Newton correction scheme, as explained below. 
3. Adaptive Newton continuation. When it comes to the numerical solution of (2.19) by means of the ordinary Newton method

$$
\begin{aligned}
\mathbf{G}^{\prime}\left(\mathbf{z}^{(\ell)} ; t_{k+1}\right) \mathbf{z}^{(\ell)} & =-\mathbf{G}\left(\mathbf{z}^{(\ell)} ; t_{k+1}\right), \\
\mathbf{z}^{(\ell+1)} & =\mathbf{z}^{(\ell)}+\Delta \mathbf{z}^{(\ell)},
\end{aligned}
$$

it turns out that its naive application is seldom successful. The reason is that the IB scheme is typically badly conditioned in the sense that even small perturbations of $\mathbf{X}$ from its target position (here: $\mathbf{X}\left(t_{k+1}\right)$ ) cause strong counterforces that lead to rapid oscillations in all unknowns resulting in non-convergence of (3.1a),(3.1b). A remedy for this problem is to use a modified Newton method with adaptively chosen damping parameters (step lengths) $\alpha_{\ell} \in\left[\alpha_{\min }, 1\right]$ for the update $\mathbf{z}^{(\ell+1)}=\mathbf{z}^{(\ell)}+\alpha_{\ell} \boldsymbol{\Delta} \mathbf{z}^{(\ell)}$. More precisely, we use the algorithm NLEQ-ERR from [15] which employs a combination of damped ordinary and simplified Newton steps in order to build certain contraction factors serving as convergence monitoring quantities (for details, see [15] and below). The actual motivation for this algorithm is that it can be used in the context of parameter-dependent nonlinear problems.

Our approach is based on the affine invariant convergence theory for Newton-like methods developed in [15] and updates the time parameter $t$ in an adaptive way using local information about the trajectory $\{\mathbf{z}(t)=(\mathbf{u}(t), \mathbf{p}(t), \mathbf{X}(t)) \mid t \in[0, T]\}$. We follow this trajectory in a predictor-corrector manner. For the computation of the prediction step it is essential how to choose the increment $\tau_{k}$, since this is closely related to the problem of staying inside the Kantorovich neighborhood of the solution at time $t_{k}+\tau_{k}$. Predictor-corrector methods can be seen as discrete continuation methods where the continuation step plays the role of a prediction step. Consider a parameter-dependent nonlinear system

$$
\mathbf{G}(\mathbf{z} ; t)=\mathbf{0}, \quad t \in[0, T], \mathbf{z} \in \mathbb{R}^{N},
$$

where $\mathbf{G}(\cdot ; t): \mathbb{R}^{N} \rightarrow \mathbb{R}^{N}$ and assume that there exists a solution $\mathbf{z}(t) \in \mathbb{R}^{N}$ for $t \in[0, T]$. Discrete continuation methods solve (3.2) with respect to a partitioning $t_{0}<t_{1}<\cdots<t_{M}=T, M>1$, of the interval $[0, T]$. This partitioning is not given in advance, but computed adaptively during the solution process. In order to solve the subproblems (2.19) successively by a Newton solver, we need sufficiently good initial guesses $\hat{\mathbf{z}}^{(0)}\left(t_{k+1}\right)$ which have to be provided by a continuation step. It has to be adapted in such a way that the convergence requirements of the subsequent correction step (2.19) are met. The quality of approximation of the prediction can be controlled by two factors, the steplength $\tau_{k}$ and the type of continuation. The simplest possible choice for a continuation step $\mathbf{z}\left(t_{k}\right) \rightarrow \hat{\mathbf{z}}\left(t_{k+1}\right)$ is constant continuation, i.e.,

$$
\hat{\mathbf{z}}\left(t_{k+1}\right):=\mathbf{z}\left(t_{k}\right)
$$

also called classical continuation. It involves no further computations and for simplicity, we will stick to this choice. By Taylor expansion, we get as approximation error

$$
\left\|\hat{\mathbf{z}}\left(t_{k}+\tau_{k}\right)-\mathbf{z}\left(t_{k}+\tau_{k}\right)\right\| \leq \eta \tau_{k}
$$

We remark that the constant $\eta=\max _{t \in[0, T]}\|\dot{z}(t)\|$ is a key quantity for an adaptive stepsize selection. It will be used below. 
The term affine covariance means that the Newton iterates $\mathbf{z}^{(\ell)}$ are invariant with respect to affine transformations $\mathbf{G} \rightarrow \mathbf{T} \mathbf{G}$ of the image space of $\mathbf{G}$ by a regular matrix $\mathbf{T}$, which can be easily seen. Invariance with respect to affine transformations of the domain space is referred to as affine contravariance. Both are subtopics to the generic term affine invariance. We encounter affine covariance in Lipschitz conditions like

$$
\left\|\left(\mathbf{G}^{\prime}\left(\mathbf{z}_{1} ; t_{k+1}\right)\right)^{-1}\left(\mathbf{G}\left(\mathbf{z}_{1} ; t_{k+1}\right)-\mathbf{G}\left(\mathbf{z}_{2} ; t_{k+1}\right)\right)\right\| \leq \omega\left\|\mathbf{z}_{1}-\mathbf{z}_{2}\right\|
$$

through its affine covariant constant. They appear in certain affine covariant Newton convergence theorems (see Theorem 3.1 below). By nature of the affine covariant concept, such theorems can only be about the iterates' increments $\boldsymbol{\Delta} \mathbf{z}^{(\ell)}$ or errors $\mathbf{z}^{(\ell)}-\mathbf{z}^{*}$, not about residuals $\mathbf{G}\left(\mathbf{z}^{(\ell)} ; t_{k+1}\right)$. This has to be taken into account in the subalgorithms of an algorithmic realization of a discrete continuation method formulated in affine covariant terms.

In our case, the object of interest is the homotopy path $\mathbf{z}(t)$ which lives in the domain of the mapping $\mathbf{G}$. Therefore, the concept of affine covariance is the adequate framework.

We consider a convergence analysis of the simplified Newton method which is most appropriate to derive steplength criteria for a discrete continuation method. We recall that for a given start iterate $\mathbf{z}^{(0)}$ the simplified Newton method is of the form

$$
\begin{aligned}
\mathbf{G}^{\prime}\left(\mathbf{z}^{(0)} ; t_{k+1}\right) \overline{\Delta \mathbf{z}}^{(\ell)} & =-\mathbf{G}\left(\mathbf{z}^{(\ell)} ; t_{k+1}\right), \\
\mathbf{z}^{(\ell+1)} & =\mathbf{z}^{(\ell)}+\overline{\boldsymbol{\Delta}}^{(\ell)}, \ell=0,1, \ldots .
\end{aligned}
$$

It is characterized by the fixed Jacobian $\mathbf{G}^{\prime}\left(\mathbf{z}^{(0)} ; t_{k+1}\right)$ which is used for all iterations. We cite the following affine covariant convergence result (cf. Theorem 2.5) in [15]):

Theorem 3.1. Let $D \subset \mathbb{R}^{N}$ be open and convex, $\mathbf{G}: D \rightarrow \mathbb{R}^{N}$ be continuously differentiable, and $\mathbf{z}^{(0)} \in D$ such that $\mathbf{G}^{\prime}\left(\mathbf{z}^{(0)}\right)$ is invertible. Further, for some $\omega>0$ let the affine covariant Lipschitz condition

$$
\left\|\left(\mathbf{G}^{\prime}\left(\mathbf{z}^{(0)}\right)\right)^{-1}\left(\mathbf{G}^{\prime}(\mathbf{z})-\mathbf{G}^{\prime}\left(\mathbf{z}^{(0)}\right)\right)\right\| \leq \omega\left\|\mathbf{z}-\mathbf{z}^{(0)}\right\|, \mathbf{z} \in D,
$$

hold true. Assume further

$$
h_{0}:=\omega\left\|\overline{\Delta \mathbf{z}}^{(0)}\right\| \leq \frac{1}{2}
$$

and define $\delta^{*}:=1-\sqrt{1-2 h_{0}}, \rho:=\delta^{*} / \omega$. Suppose that the closed ball $\overline{B_{\rho}\left(\mathbf{z}^{(0)}\right)}$ is contained in $D$.

Then, the simplified Newton method (3.5) generates iterates $\left\{\mathbf{z}^{(\ell)}\right\}_{\ell \in \mathbb{N}} \subset \overline{B_{\rho}\left(\mathbf{z}^{(0)}\right)}$ converging to $\mathbf{z}^{*}$ with $\mathbf{G}\left(\mathbf{z}^{*}\right)=0$, and there holds the estimate

$$
\frac{\left\|\mathbf{z}^{(\ell+1)}-\mathbf{z}^{(\ell)}\right\|}{\left\|\mathbf{z}^{(\ell)}-\mathbf{z}^{(\ell-1)}\right\|} \leq \frac{1}{2}\left(\delta_{\ell}+\delta_{\ell-1}\right), \quad \ell \geq 1,
$$

where $\delta_{0}:=0$ and $\delta_{\ell+1}:=h_{0}+1 / 2 \delta_{\ell}^{2}, \ell \geq 0$.

The contraction factors

$$
\Theta_{\ell}:=\frac{\left\|\overline{\Delta \mathbf{z}}^{(\ell+1)}\right\|}{\left\|\overline{\Delta \mathbf{z}}^{(\ell)}\right\|}, \ell \geq 0
$$


serve as monitoring quantities in Newton algorithms: From the convergence rate estimate (3.8) it follows that $\Theta_{\ell} \leq 1 / 2\left(\delta_{\ell+1}+\delta_{\ell}\right), \ell \geq 0$. Along with $\delta_{0}=0, \delta_{1}=h_{0}$ and the definition of $h_{0}$ from (3.7), we get

$$
\Theta_{0} \leq \frac{1}{2} \omega\left\|\overline{\mathbf{\Delta z}}^{(0)}\right\|
$$

This estimate is equivalent to

$$
\frac{2 \Theta_{0}}{\left\|\overline{\boldsymbol{\Delta}}^{(0)}\right\|} \leq \omega
$$

which provides a lower bound for $\omega$ respectively the local Lipschitz constant $\widehat{\omega}$ (cf. Theorem 3.2 below). Along with $\eta$ from (3.4), this is the second key quantity for an adaptive step size selection strategy. In the following, we have to assume that $\tau \geq \tau^{\mathrm{min}}$ for some given $\tau^{\mathrm{min}}>0$ to guarantee boundedness of $\gamma^{-1}$ (cf. (2.24)). This is in agreement with the algorithmic realization of the predictor-corrector scheme (see Algorithm 3.6 below) where the case $\tau_{k}<\tau^{\mathrm{min}}$ serves as a convergence failure criterion.

TheOrem 3.2. Let $\mathbf{G}^{\prime}(\mathbf{z} ; t)$ be nonsingular for all $(\mathbf{z} ; t) \in D \times[0, T]$, assume that the homotopy path $\mathbf{z}:[0, T] \rightarrow D$ exists, and let $t_{k} \in[0, T]$. Further, suppose that there exists a local Lipschitz constant $\widehat{\omega}$ such that for all $\mathbf{z} \in D$ and for all $0<\tau^{\min } \leq \tau \leq T-t_{k}$ satisfying $\hat{\mathbf{z}}\left(t_{k}+\tau\right) \in D$, the affine covariant Lipschitz condition

$$
\begin{aligned}
& \left\|\left(\mathbf{G}^{\prime}\left(\hat{\mathbf{z}}\left(t_{k}+\tau\right) ; t_{k}+\tau\right)\right)^{-1}\left(\mathbf{G}^{\prime}\left(\mathbf{z} ; t_{k}+\tau\right)-\mathbf{G}^{\prime}\left(\hat{\mathbf{z}}\left(t_{k}+\tau\right) ; t_{k}+\tau\right)\right)\right\| \\
& \leq \widehat{\omega}\left\|\mathbf{z}-\hat{\mathbf{z}}\left(t_{k}+\tau\right)\right\|
\end{aligned}
$$

holds true. Then, the simplified Newton method (3.5) with starting point $\hat{\mathbf{z}}\left(t_{k}+\tau\right):=$ $z\left(t_{k}\right)$ (classical continuation) converges towards the solution $\mathbf{z}\left(t_{k}+\tau\right)$ for all stepsizes

$$
\tau^{\min } \leq \tau \leq \tau^{\max }\left(t_{k}\right):=\frac{\sqrt{2}-1}{\widehat{\omega} \eta}
$$

Proof. For completeness we present the proof from Corollary 5.5 in [15], since we will use an estimate from the proof for the subsequent discussion. For the application of Theorem 3.1 we need

$$
\widehat{\omega}\left\|\overline{\boldsymbol{\Delta}}^{(0)}\left(t_{k}+\tau\right)\right\| \leq \frac{1}{2} .
$$

To this end, we derive an upper bound $r(\tau)$ for $\left\|\overline{\mathbf{\Delta z}}^{(0)}\right\|$. We set $\hat{\mathbf{z}}:=\hat{\mathbf{z}}\left(t_{k+1}\right)$, 
$\mathbf{z}:=\mathbf{z}\left(t_{k+1}\right)$ and consider

$$
\begin{aligned}
& \left\|\overline{\Delta \mathbf{z}}^{(0)}\left(t_{k+1}\right)\right\| \\
& =\left\|\left(\mathbf{G}^{\prime}\left(\hat{\mathbf{z}}, t_{k+1}\right)\right)^{-1} \mathbf{G}\left(\hat{\mathbf{z}}, t_{k+1}\right)\right\|=\left\|\left(\mathbf{G}^{\prime}\left(\hat{\mathbf{z}} ; t_{k+1}\right)\right)^{-1}(\mathbf{G}\left(\hat{\mathbf{z}} ; t_{k+1}\right)-\overbrace{\mathbf{G}\left(\mathbf{z} ; t_{k+1}\right)}^{=\mathbf{0}})\right\| \\
& =\left\|\left(\mathbf{G}^{\prime}\left(\hat{\mathbf{z}}, t_{k+1}\right)\right)^{-1} \int_{0}^{1} \mathbf{G}^{\prime}\left(\mathbf{z}+s(\hat{\mathbf{z}}-\mathbf{z}) ; t_{k+1}\right)(\hat{\mathbf{z}}-\mathbf{z}) d s\right\| \\
& =\left\|\int_{0}^{1}\left(\mathbf{G}^{\prime}\left(\hat{\mathbf{z}} ; t_{k+1}\right)\right)^{-1}\left(\mathbf{G}^{\prime}\left(\mathbf{z}+s(\hat{\mathbf{z}}-\mathbf{z}), t_{k+1}\right) \pm \mathbf{G}^{\prime}\left(\hat{\mathbf{z}}, t_{k+1}\right)\right)(\hat{\mathbf{z}}-\mathbf{z}) d s\right\| \\
& \leq\|\hat{\mathbf{z}}-\mathbf{z}\|\left(1+\int_{0}^{1}\left\|\left(\mathbf{G}^{\prime}\left(\hat{\mathbf{z}} ; t_{k+1}\right)\right)^{-1}\left(\mathbf{G}^{\prime}\left(\mathbf{z}+s(\hat{\mathbf{z}}-\mathbf{z}) ; t_{k+1}\right)-\mathbf{G}^{\prime}\left(\hat{\mathbf{z}} ; t_{k+1}\right)\right)\right\| d s\right) \\
& \stackrel{(3.12)}{\leq}\|\hat{\mathbf{z}}-\mathbf{z}\|\left(1+\widehat{\omega}\|\hat{\mathbf{z}}-\mathbf{z}\| \int_{0}^{1}|1-s| d s\right) .
\end{aligned}
$$

From the definition of $\eta(\operatorname{cf} .(3.4))$ we know $\left\|\hat{\mathbf{z}}\left(t_{k+1}\right)-\mathbf{z}\left(t_{k+1}\right)\right\| \leq \eta \tau$, whence

$$
\left\|\overline{\boldsymbol{\Delta}}^{(0)}\left(t_{k}+\tau\right)\right\| \leq \eta \tau\left(1+\frac{1}{2} \widehat{\omega} \eta \tau\right)=: r(\tau)
$$

The crucial step is to use this estimate for requirement (3.14) to arrive at

$$
\widehat{\omega} \eta \tau\left(1+\frac{1}{2} \widehat{\omega} \eta \tau\right) \leq \frac{1}{2},
$$

which is fulfilled for $\widehat{\omega} \eta \tau \leq \sqrt{2}-1$. Under this condition, which is equivalent to (3.13), Theorem 3.1 can be applied, and we conclude.

REMARK 3.3. According to Theorem 3.2, the iterates $\left\{\mathbf{z}^{(\ell)}\right\}_{\ell}$ stay within the closed ball $\overline{B_{\rho}\left(\hat{\mathbf{z}}^{(0)}\left(t_{k+1}\right)\right)} \subset \overline{B_{1 / \hat{\omega}}\left(\mathbf{z}\left(t_{k}\right)\right)}=: B$, if the step size restriction $(3.13)$ is fulfilled. (This is also true when, in practice, we use a combination of the ordinary and the simplified Newton method.) Hence, all Newton iterates are well defined, if in view of (2.22) we choose

$$
\tau^{\min } \leq \tau_{k} \leq \min \left(\frac{\sqrt{2}-1}{\widehat{\omega} \eta}, \min _{\mathbf{z} \in B}\left(\tau^{\max }(\mathbf{z})\right)\right)
$$

REMARK 3.4. We check the affine covariant Lipschitz condition (3.12) from Theorem 3.2 in case the nonlinear mapping $\mathbf{G}\left(\cdot ; t_{k+1}\right), t_{k+1}=t_{k}+\tau, k \in \mathbb{N}_{0}$, is given by (2.20). Let $\tau \geq \tau^{\mathrm{min}}>0$ as in Theorem 3.2. Setting for brevity $\hat{\mathbf{z}}:=\hat{\mathbf{z}}\left(t_{k}+\tau\right)$, we have

$$
\begin{aligned}
& \mathbf{G}^{\prime}\left(\mathbf{z} ; t_{k}+\tau\right)-\mathbf{G}^{\prime}\left(\hat{\mathbf{z}}\left(t_{k}+\tau\right) ; t_{k}+\tau\right)= \\
& \left(\begin{array}{ccc}
\tau\left(\mathbf{C}^{\prime}(\mathbf{u})-\mathbf{C}^{\prime}(\hat{\mathbf{u}})\right) & \mathbf{0} & -\tau\left(\mathbf{F}^{\prime}(\mathbf{X})-\mathbf{F}^{\prime}(\hat{\mathbf{X}})\right) \\
\mathbf{0} & \mathbf{0} & \mathbf{0} \\
-\tau(\mathbf{K}(\mathbf{X})-\mathbf{K}(\hat{\mathbf{X}})) & \mathbf{0} & -\tau\left(\mathbf{K}_{\mathbf{X}}^{\prime}(\mathbf{X}, \mathbf{u})-\mathbf{K}_{\mathbf{X}}^{\prime}(\hat{\mathbf{X}}, \hat{\mathbf{u}})\right)
\end{array}\right)
\end{aligned}
$$


We denote by $L_{\mathbf{C}^{\prime}}$ the (local) Lipschitz constant $L_{\mathbf{C}^{\prime}}\left(\mathbf{z}\left(t_{k}\right)\right)$ of the Fréchet derivative $\mathbf{C}^{\prime}$, i.e.,

$$
\left\|\mathbf{C}^{\prime}(\mathbf{u})-\mathbf{C}^{\prime}(\hat{\mathbf{u}})\right\| \leq L_{\mathbf{C}^{\prime}}\|\mathbf{u}-\hat{\mathbf{u}}\|
$$

and define $L_{\mathbf{F}^{\prime}}, L_{\mathbf{K}}, L_{\mathbf{K}_{\mathbf{x}}^{\prime}}$ similarly. Then, with $\gamma=\gamma\left(\mathbf{z}\left(t_{k}\right)\right)$ and in view of $(2.24)$, from Theorem 2.3 we obtain (3.12) with a $\tau$-independent upper bound

$$
\widehat{\Omega}:=\gamma^{-1}\left(T-t_{k}\right) \max \left(L_{\mathbf{C}^{\prime}}, L_{\mathbf{F}^{\prime}}, L_{\mathbf{K}}, L_{\mathbf{K}_{\mathbf{X}}^{\prime}}\right) .
$$

The result of the last theorem has the drawback that it contains the unknown constants $\widehat{\omega}$ and $\eta$. In order to exploit the steplength criterion (3.13) in an algorithmic realization, we use the following strategy:

We compute estimates [.] of the a priori unknown constants and apply the steplength criterion with $\widehat{\omega}, \eta$ replaced with $[\widehat{\omega}],[\eta]$. By nature of the constants $\widehat{\omega}, \eta$ we have to work with lower bounds. Taking into account that both quantities appear in the denominator of (3.13), this strategy will in general overestimate the true maximal steplength $\tau^{\max }\left(t_{k}\right)$. Therefore, we only predict a maximal stepsize based on some estimates of the local constant $\widehat{\omega}$ and the global constant $\eta$. It explains why we also need a correction formula for $\tau_{k}$ in case of convergence failure of the Newton corrector.

REMARK 3.5. There is some ambiguity of the terms 'prediction' and 'correction', since we use each of them in two different meanings:

(a) In the context of a continuation method, we usually mean the prediction and the correction of the variable $\mathbf{z}$. Therefore, we use the expressions $\mathbf{z}$-prediction (by classical continuation) and $\mathbf{z}$-correction (by a Newton solver), respectively. For the continuation step in the $\mathbf{z}$-prediction,

(b) the steplength $\tau$ is predicted and, if necessary, corrected. We will address this as $\tau$-prediction and $\tau$-correction, respectively.

3.1. $\tau$-prediction strategy. We can use estimate (3.11) to obtain

$$
\frac{2 \Theta_{0}\left(t_{k}\right)}{\left\|\overline{\boldsymbol{\Delta z}}^{(0)}\left(t_{k}\right)\right\|}=:[\widehat{\omega}] \leq \widehat{\omega}
$$

as an estimate for $\widehat{\omega}$ and likewise

$$
\frac{\left\|\hat{\mathbf{z}}\left(t_{k}\right)-\mathbf{z}\left(t_{k}\right)\right\|}{\tau_{k-1}}=:[\eta] \leq \eta
$$

which obviously provides a lower bound of $\eta$ due to (3.4). Inserting these estimates (which have the advantage of being computationally available) into (3.13) instead of $\widehat{\omega}$ and $\eta$, results in the $\tau$-prediction formula

$$
\tau_{k}:=\tau_{k, 0}:=\left[\tau^{\max }\left(t_{k}\right)\right]:=\frac{(\sqrt{2}-1)\left\|\mathbf{\Delta z}^{(0)}\left(t_{k}\right)\right\|}{2 \Theta_{0}\left(t_{k}\right)\left\|\mathbf{z}^{(0)}\left(t_{k}\right)-\mathbf{z}\left(t_{k}\right)\right\|} \tau_{k-1} .
$$

This formula predicts the next steplength $\tau_{k}$ adaptively based upon information about local and global constants of the homotopy gathered within the last Newton correction step. In (3.16) we have used the fact that the first increment of the simplified and the ordinary Newton method coincide. Since the exact solution $\mathbf{z}\left(t_{k}\right)$ is not known in general, we use the final solution of the Newton corrector as its replacement in (3.16) to obtain a computable expression. Due to the fact that this formula defines $\tau_{k}$ recursively, we need to specify some $\tau_{0}$ for the first continuation step. 
3.2. $\tau$-correction strategy. From (3.16) it is clear that $\left[\tau^{\max }\left(t_{k}\right)\right] \geq \tau^{\max }\left(t_{k}\right)$, and so the predicted steplength $\tau_{k}=\tau_{(k, 0)}$ may be too large and could lead to a convergence failure of the Newton correction step. In this case, we need to correct it, i.e., $\tau_{(k, j+1)} \leftarrow \tau_{(k, j)}$ and repeat the last $\mathbf{z}$-prediction step, now with an adaptively reduced stepsize $\tau_{k, j+1}$. Here, we can deal with the quantity $\Theta_{0}\left(t_{k}+\tau_{(k, j)}\right)$, since we have computed it in the last unsuccessful Newton correction at $t_{k}+\tau_{(k, j)}$. It can be exploited to gain refined information about the crucial quantity $\widehat{\omega} \eta$ from (3.13): Estimate (3.10) in combination with (3.15) gives (using $\left.t_{(k+1, j)}:=t_{k}+\tau_{(k, j)}\right)$ :

$$
\Theta_{0}\left(t_{(k+1, j)}\right) \leq \frac{1}{2} \widehat{\omega} \eta \tau_{(k, j)}\left(1+\frac{1}{2} \widehat{\omega} \eta \tau_{(k, j)}\right)
$$

Since $\widehat{\omega}, \eta$, and $\tau_{(k, j)}$ are all nonnegative quantities, this is equivalent to

$$
\widehat{\omega} \eta \tau_{(k, j)} \geq \sqrt{4 \Theta_{0}\left(t_{(k+1, j)}\right)+1}-1 \Longleftrightarrow \widehat{\omega} \eta \geq \underbrace{\frac{\sqrt{4 \Theta_{0}\left(t_{(k+1, j)}\right)+1}-1}{\tau_{(k, j)}}}_{=:[\widehat{\omega} \eta]} .
$$

Plugging this lower bound for $\widehat{\omega} \eta$ into the stepsize formula (3.13), we obtain the $\tau$-correction formula:

$$
\tau_{(k, j+1)}:=\left[\tau^{\max }\left(t_{k}\right)\right]:=\frac{\sqrt{2}-1}{\sqrt{4 \Theta_{\ell(j)}\left(t_{(k+1, j)}\right)+1}-1} \tau_{(k, j)}, j \geq 0 .
$$

Here, $\Theta_{0}$ has been replaced with $\Theta_{\ell(j)}$, where $\ell(j)$ stands for the $\ell$-th Newton iteration (in the $j$-th $\tau$-correction cycle, $j \geq 0$ ) where convergence failure has occurred. This is not backed by rigorous theory but seems unavoidable and reasonable to get an executable algorithm:

Convergence failure for the simplified Newton method is accompanied by $\Theta_{\ell(j)} \geq 1$ (and nevertheless, if $\ell(j) \geq 1$ it is possible that $1 / 4<\Theta_{0(j)}<1$ ! $\Theta_{0} \leq 1 / 4$ implies local convergence of the simplified Newton method, see (3.7) and (3.10)). Similarly, using the algorithm NLEQ-ERR as a Newton corrector, for convergence failure we must have $\Theta_{\ell(j)} \geq 1$. In both cases, for $\Theta_{\ell(j)} \geq 1$ we get an obvious reduction of the steplength, since

$$
\frac{\sqrt{2}-1}{\sqrt{4 \Theta_{\ell(j)}\left(t_{(k+1, j)}\right)+1}-1} \leq \frac{\sqrt{2}-1}{\sqrt{5}-1} \approx 0.34 \text {. }
$$

3.3. Adaptive continuation algorithm. We summarize the discussion about the time increment-adaptive prediction-correction scheme by the following pseudocode. The reader may concentrate, in particular, on the $\tau$-prediction-correction for a clarification of the preceding discussion.

\footnotetext{
Algorithm 3.6. Adaptive predictor-corrector algorithm

$\%$ Initialization:

Specify the initial IB state variables $\left(\mathbf{u}^{(0)}, \mathbf{p}^{(0)}, \mathbf{X}^{(0)}\right)=: \mathbf{z}\left(t_{0}\right)$ and a starting value $\tau_{(0,0)}$, bounds $\tau^{\min }$ and $\tau^{\max }$, and $\Theta_{\min } \ll 1$.

Set $t_{0}:=0, k:=0$, and $j:=0$.
} 
$\%$ Iteration:

while $t_{k}<T$

$\% \mathbf{z}$-prediction step:

Set $t_{(k+1, j)}:=t_{k}+\tau_{(k, j)}$

Perform the classical continuation step $\hat{\mathbf{z}}^{(0)}\left(t_{k+1}\right):=\mathbf{z}\left(t_{k}\right)$

$\% \mathbf{z}$-correction step:

Solve $\mathbf{G}\left(\mathbf{z} ; t_{k+1}\right)=0$ with initial guess $\hat{\mathbf{z}}^{(0)}\left(t_{k+1}\right)$ by the Newton solver NLEQ-ERR: Thereby, contraction factors $\Theta_{\ell}=\Theta_{\ell}\left(t_{(k+1, j)}\right), \ell \geq 0$, are computed and $\mathbf{z}$ gets updated by means of a damping factor $\alpha_{\ell}$ according to $\hat{\mathbf{z}}^{(\ell+1)}\left(t_{k+1}\right)=\hat{\mathbf{z}}^{(\ell)}\left(t_{k+1}\right)+\alpha_{\ell} \boldsymbol{\Delta} \mathbf{z}^{(\ell)}$.

if Newton corrector was successful after $\ell=\ell(j) \geq 1$ iterations

Set $\mathbf{z}\left(t_{k+1}\right):=\hat{\mathbf{z}}^{(\ell)}\left(t_{k+1}\right)$

Set $t_{k+1}:=t_{(k+1, j)}$

Set $\Theta_{0}:=\max \left(\Theta_{0}, \Theta_{\min }\right)$

Predict the new time increment by

$$
\tau_{(k+1,0)}:=\frac{(\sqrt{2}-1)\left\|\Delta \mathbf{z}^{(0)}\right\|}{2 \Theta_{0}\left\|\hat{\mathbf{z}}^{(0)}\left(t_{k+1}\right)-\mathbf{z}\left(t_{k+1}\right)\right\|} \tau_{(k, j)}
$$

Set $\tau_{(k+1,0)}:=\min \left(\tau_{(k+1,0)}, \tau^{\max }\right)$

if $\tau_{(k+1,0)}<\tau^{\mathrm{min}}$, stop: convergence failure end

else

Correct $\tau$ according to

$$
\tau_{(k, j+1)}:=\frac{\sqrt{2}-1}{\sqrt{4 \Theta_{i(j)}\left(t_{(k+1, j)}\right)+1}-1} \tau_{(k, j)}
$$

if $\tau_{(k, j+1)}<\tau^{\mathrm{min}}$, stop: convergence failure end

Set $j \leftarrow j+1$ and go back to z-prediction step

end

Set $j:=0$

Set $k \leftarrow k+1$

end

4. Numerical results. As an illustration of the adaptive continuation method we consider the motion and deformation of a red blood cell (RBC) immersed in an external microfluidic channel flow passing through a thin capillary. The plasma membrane of an RBC consists of a lipid bilayer membrane and an attached spectrin network as cytoskeleton [3]. Therefore, one might expect a rather complex fluidic behavior, since the underlying cytoskeleton can rearrange according to an external mechanical force [4]. However, it was demonstrated that lipid vesicles without an attached polymer network may serve as a simple model which already captures the basic physics of the fluid-structure interaction problem. We note that the dynamics of RBC and vesicles in fluid flow has been studied both experimentally (see $[1,2,19$, $20,26,37]$ ) and theoretically (cf. [6, 7, 21, 22, 32, 34]). For the numerical study of the rheology of $\mathrm{RBC}$ in microchannels, the IB has been applied in [5, 17, 27, 38], whereas 
the FE-IB has been used recently in [16].

It is well known that an RBC can pass through capillaries whose diameters are half or even less than the typical diameter of an RBC $(7.5-8 \mu \mathrm{m})$, enabling oxygen supply also through highly branched blood vessels. We have considered a microchannel with a diameter varying between 20 and $4 \mu \mathrm{m}$, a density of $\rho=1.0 \cdot 10^{3} \mathrm{~kg} / \mathrm{m}^{3}$, and a viscosity of $\nu=6.0 \cdot 10^{-3} \mathrm{~Pa} \cdot \mathrm{s}$ both for the carrier fluid and the fluid enclosed by the membrane of the RBC. We have further assumed an inflow velocity of $g=1.0 \cdot 10^{-2} \mathrm{~m} / \mathrm{s}$. Taking $d=20 \mu \mathrm{m}$ as reference length, this results in a Reynolds number of $\mathcal{R} e \approx 3 \cdot 10^{-2}$, a typical magnitude for RBCs in microfluidic flows. We note that the maximal velocity inside the narrow part of the channel is almost five times higher than the inflow velocity which results in a strong deformation of the passing $\mathrm{RBC}$ whose elastic moduli have been chosen according to $\kappa_{e}=6.0 \cdot 10^{-6} \mathrm{~N} / \mathrm{m}$ and $\kappa_{b}=2.0 \cdot 10^{-19} \mathrm{Nm}$ (cf. [35]). In its initial state, the RBC has a diameter of $7.8 \mu \mathrm{m}$ and a perimeter of $L=19.8 \mu \mathrm{m}$. We have used a uniform finite element mesh for $\Omega=[0,50] \times[0,20] \backslash(17.5,32.5) \times$ $((0,8) \cup(12,20)) \mu \mathrm{m}^{2}$ with mesh parameter $h=1.0 \mu \mathrm{m}$ and a uniform partition of $[0, L]$, resulting in $N_{1}+N_{2}+N_{3}=10358+1340+114=11812$. All computations have been performed under Linux on a work station featuring Intel Quad-CPU with $2.83 \mathrm{GHz}$ each and $8 \mathrm{~GB}$ RAM.

We illustrate the difficulty associated with the semi-implicit BE/FE FE-IB from [16] by considering an $\mathrm{RBC}$ in a microchannel passing a thin capillary. The (constant) time step size was chosen too large, namely, $\tau=1 / 250$, and oscillations in the IB state variables occurred leading to a 'torn apart' membrane. This is shown in Figure 4.1. The same problem occurs even for $\tau=1 / 350$, which will be of interest later.

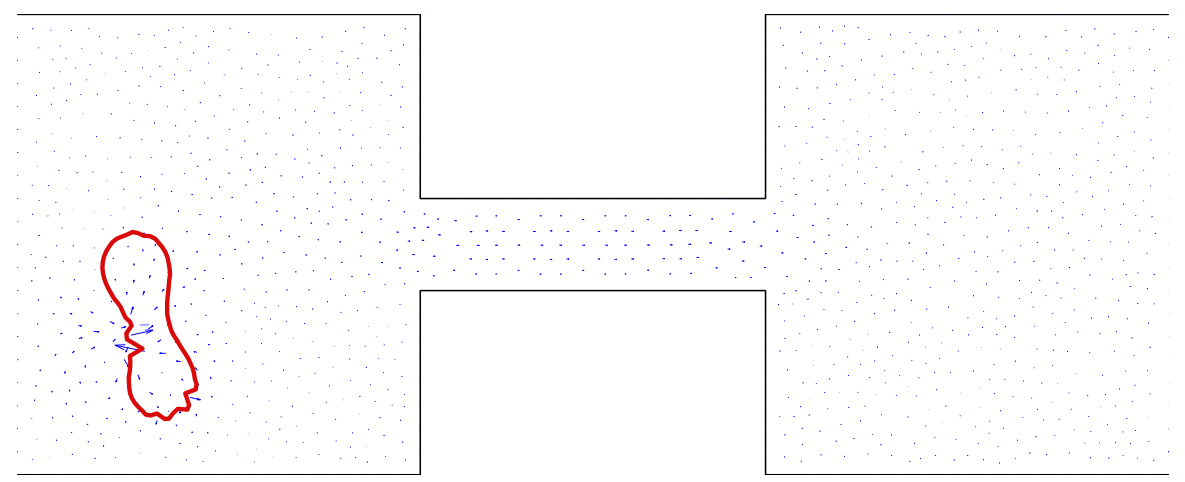

FIG. 4.1. Numerical instability of the BE/FE FE-IB for $\tau=1 / 250$. The computation was stopped at $t=0.05$ after one membrane node had left the domain (not shown in the figure) due to high oscillations.

In case of the fully implicit BE/BE FE-IB, the adaptively chosen time step sizes prevent from numerical instabilities. The adaptive step size selection algorithm detects the critical stage during the computation. This can be seen in Figure 4.2 where $\tau_{k}$ gets clearly reduced (at approximately $t=0.90$ ) and stays small as long as the RBC occupies the capillary. In the same figure, the evolution of the (scaled) total energy $E_{\text {tot }}(t)$ of the system is displayed. An increase causes a reduction of $\tau_{k}$ and vice versa: when $E_{\text {tot }}(t)$ reaches its initial standard after leaving the thin capillary, $\tau$ is chosen larger again. The time instants of the snapshots of the RBC in Figure 4.3 correspond to the markers in Figure 4.2. It is noteworthy that the average time stepsize of the 
$\mathrm{BE} / \mathrm{BE}$ FE-IB version is roughly $1 / 169$. This means that the semi-implicit version with constant time steps $\tau=1 / 350$ (see above) fails although its time increments are less than half of the average time step size of the fully implicit scheme. Moreover, in none of the $441 \mathrm{BE} / \mathrm{BE} \mathrm{FE}-\mathrm{IB}$ time steps it was necessary to perform a $\tau$-correction, which seems to indicate that the $\tau$-prediction mechanism is quite reliable.

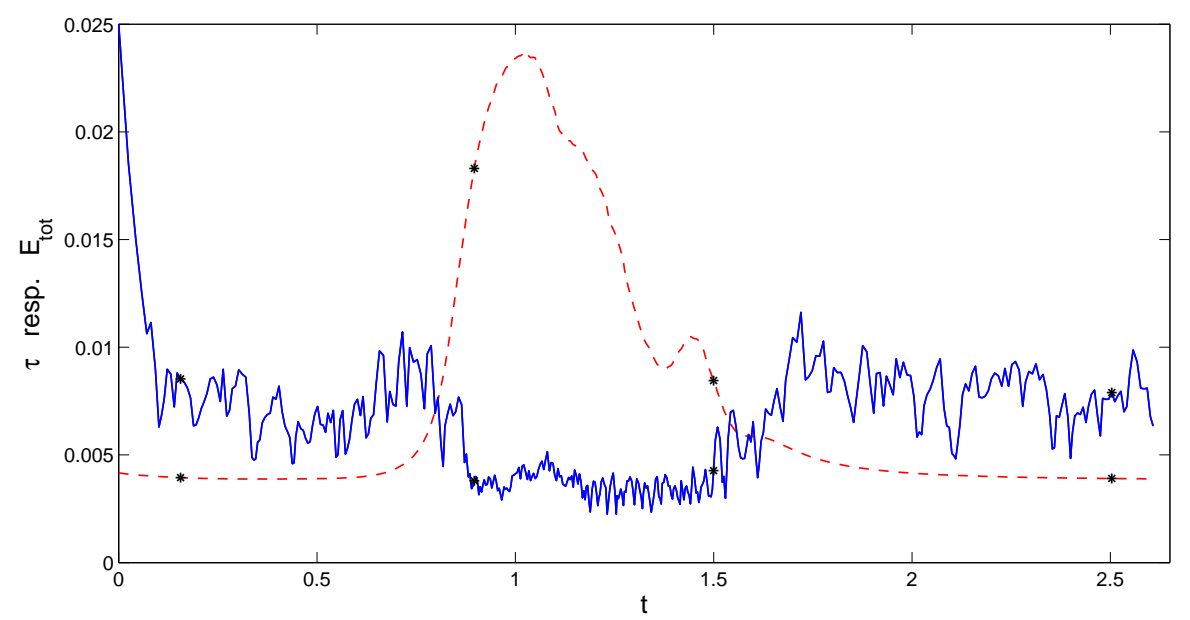

FIG. 4.2. Evolution of the adaptively chosen time increments (solid line) and of the (scaled) total energy of the system (dashed line).

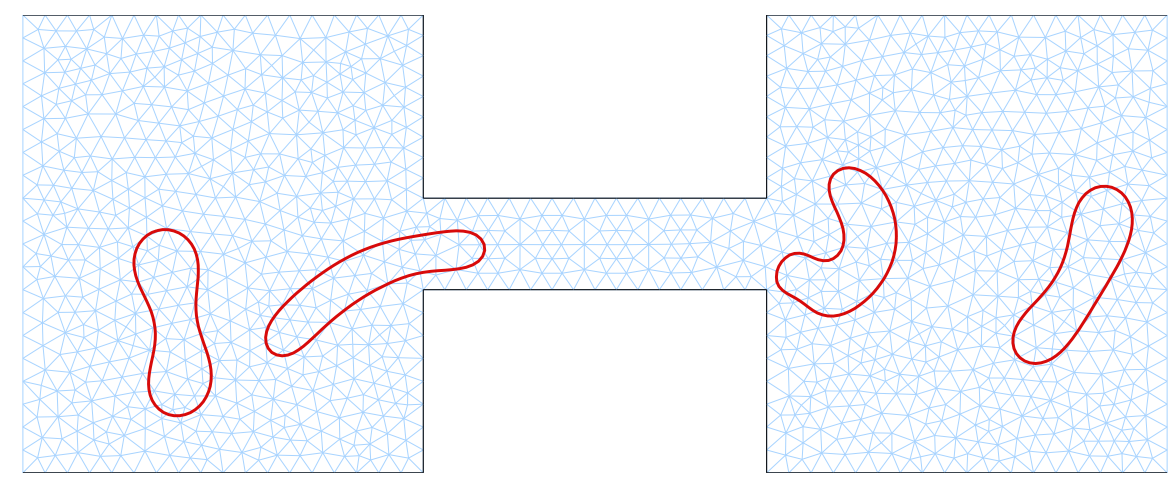

FIG. 4.3. Snapshots of the RBC's membrane at selected time instants. These time instants correspond to the *-marked instants in Figure 4.2.

The computation was stopped at $T=2.61$ shortly before the RBC reached the outflow boundary. The computational time for the fully implicit BE/BE FE-IB with initial $\tau_{(0,0)}=1 / 40$ was 2 h 25 min.

\section{REFERENCES}

[1] M. Abkarian, C. Lartigue, and A. Viallat; Tank treading and unbinding of deformable vesicles in shear flow: Determination of the lift force. Phys. Rev. Lett 88, 068103, 2002

[2] M. Abkarian and A. Viallat; Dynamics of vesicles in a wall-bounded shear flow. Biophys.J.89, 1055,2005 
[3] B. Alberts, A. Johnson, J. Lewis, M. Raff, K. Roberts, und P. Walter; Molekularbiologie der Zelle. Wiley-VCH, Weinheim, 2004.

[4] X. An, M.C. Lecomte, J.A. Chasis, N. Mohandas, and W. Gratzer; Shear-response of the spectrin dimer-tetramer equilibrium in the red blood cell membrane. J. Biol. Chem. 277, 35, 31796-31800, 2002

[5] P. Bagchi, P. Johnson, and A. Popel; Computational fluid dynamic simulation of aggrgation of deformable cells in a shear flow. J. Biomech. Eng. 127, 1070-1080, 2005

[6] J. Beaucourt, F. Rioual, T. Seon, T. Biben, and C. Misbah; Steady to unsteady dynamics of a vesicle in a flow. Phys. Rev. E 69, 011906, 2004

[7] T. Biben and C. Misbah; Tumbling of vesicles under shear flow within an advected-field approach. Phys. Rev. E 67, 031908, 2003

[8] D. Boffi and L. Gastaldi; A finite element approach for the immersed boundary method. Comput. Struct. 81, 491-501, 2003

[9] D. Boffi, L. Gastaldi, and L. Heltai; Numerical stability of the finite element immersed boundary method. Math. Mod. Meth. Appl. Sci. 17, 1479-1505, 2007

[10] D. Boffi, L. Gastaldi, and L. Heltai; On the CFL condition for the finite element immersed boundary method. Computers \& Structures 85, 775-783, 2007

[11] D. Boffi, L. Gastaldi, L. Heltai, and C. Peskin; On the hyper-elastic formulation of the immersed boundary method. Comput. Meth. in Appl. Mech. Engrg. 197, 2210-2231, 2008

[12] D. Braess; Finite Elements: Theory, Fast Solvers, and Applications in Solid Mechanics. Cambridge University Press, 3rd edition, 2007.

[13] S.C. Brenner and R. Scott, The Mathematical Theory of Finite Element Methods. 3rd Edition. Springer, Berlin-Heidelberg-New York, 2008

[14] F. Brezzi and M. Fortin; Mixed and Hybrid Finite Element Methods. Springer, BerlinHeidelberg-New York, 1991

[15] P. Deuflhard, Newton Mehtods for Nonlinear Problems - Affine Invariance and Adaptive Algorithms. Springer, Berlin-Heidelberg-New York, 2004

[16] T. Franke, R.H.W. Hoppe, C. Linsenmann, L. Schmid, C. Willbold, and A. Wixforth, Numerical simulation of the motion and deformation of red blood cells and vesicles in microfluidic flows. submitted to Computing and Visualization in Science, 2010

[17] C.D. Eggleton and A.S. Popel; Large deformation of red blood cell ghosts in simple shear flow. Physics of Fluids 10, 1834-1845, 1998

[18] P. Grisvard; Elliptic Problems in Nonsmooth Domains. Pitman, Boston, 1985

[19] K. de Haas, C. Bloom, D. van den Ende, M. Duits, and J. Mellema ; Deformation of giant lipid bilayer vesicles in shear flow. Phys. Rev. E 56, 7132, 1997

[20] V. Kantsler and V. Steinberg; Orientation and dynamics of a vesicle in tank-treading motion in shear flow. Phys. Rev. Lett. 95, 258101, 2005

[21] S. R. Keller and R. Skalak; Motion of a tank-treading ellipsoidal particle in a shear flow. J. Fluid Mech. 120, 27, 1982

[22] M. Kraus, W. Wintz, U. Seifert, and R. Lipowsky; Fluid vesicles in shear flow. Phys. Rev. Lett. 77, 3685, 1996

[23] D.V. Le, J. White, J. Peraire, K.M. Lim, and B.C. Khoo, An implicit immersed boundary method for three-dimensional fluid-membrane interactions. J. Comput. Phys. 228, 84278445,2009

[24] P. Lee, B.E. Griffith, and C.S. Peskin, The immersed boundary method for advectionelectrodiffusion with implicit timestepping and local mesh refinement. J. Comput. Phys. 229, 5208-5227, 2010

[25] Y. Mori and C.S. Peskin, Implicit second-order immersed boundary methods with boundary mass. Comput. Methods Appl. Mech. Engrg. 197, 2049-2067, 2008

[26] H. Noguchi, G. Gompper, L. Schmid, A. Wixforth, and T. Franke; Dynamics of fluid vesicles in flow through structured microchannels. Eur. Phys. Lett. (in press), 2010

[27] T.-W. Pan and T. Wang; Dynamical simulation of red blood cell rheology in microvessels. Int. J. Numer. Anal. Modeling 6, 455-473, 2009

[28] C. Peskin; Numerical analysis of flood flow in the heart. J. Comput. Phys. 25, 220-252, 1977.

[29] C. Peskin; The immersed boundary method. Acta Numerica 11, 479-517, 2002.

[30] C. Peskin and D.M. McQueen; A three-dimensional computational method for blood flow in the heart. I. Immersed elastic fibers in a viscous incompressible fluid. J. Comput. Phys. 81, 372-405, 1989.

[31] C. Peskin and B.F Printz; Improved volume conservation in the computation of flows with immersed elastic boundaries. J. Comput. Phys. 105, 33-46, 1993.

[32] F. Rioual, T. Biben, and C. Misbah; Analytical analysis of a vesicle tumbling under a shear flow. Phys. Rev. E 69, 061914, 2004 
[33] M.E. Rosar and C.S. Peskin; Fluid flow in collapsible elastic tubes: A three-dimensional numerical model. New York J. Math. 7, 281-302, 2001

[34] U. Seifert; Fluid membranes in hydrodynamic flow fields: Formalism and an application to fluctuating quasispherical vesicles in shear flow. Eur. Phys. J. B 8, 405, 1999

[35] R. Skalak and S. Chien; Handbook of Bioengineering. McGraw-Hill, New York, 1987

[36] L. Tartar; Introduction to Sobolev Spaces and Interpolation Theory. Springer, BerlinHeidelberg-New York, 2007

[37] V. Vitkova, M. Mader, T. Biben, and T. Podgorski; Tumbling of lipid vesicles, enclosing a viscous fluid, under a shear flow. J. Optoelectr. Adv. Mater. 7, 261, 2005

[38] T. Wang, T.-W. Pan, Z.W. Xing, and R. Glowinski; Numerical simulation of red blood cell rouleaus in microchannels. Phys. Rev. E 79, 041916-1 - 041916-11, 2009 